Article

\title{
Optimisation of Milk Protein Top-Down Sequencing Using In-Source Collision-Induced Dissociation in the Maxis Quadrupole Time-of-Flight Mass Spectrometer
}

\author{
Delphine Vincent ${ }^{1, *}$, Dominik Mertens ${ }^{2}$ and Simone Rochfort ${ }^{1,3}$ \\ 1 Department of Economic Development, Jobs, Transport and Resources, AgriBio Centre, Bundoora, \\ Victoria 3083, Australia; simone.rochfort@ecodev.vic.gov.au \\ 2 Genedata AG, 4016 Basel, Switzerland; Dominik.Mertens@genedata.com \\ 3 School of Applied Systems Biology, La Trobe University, Bundoora, Victoria 3083, Australia \\ * Correspondence: delphine.vincent@ecodev.vic.gov.au; Tel.: +61-3-9032-7116
}

Academic Editor: Paolo Iadarola

Received: 8 October 2018; Accepted: 25 October 2018; Published: 26 October 2018

\begin{abstract}
Top-down sequencing in proteomics has come of age owing to continuous progress in LC-MS. With their high resolution and broad mass range, Quadrupole Time-of-Flight (Q-ToF) hybrid mass spectrometers equipped with electrospray ionisation source and tandem MS capability by collision-induced dissociation (CID) can be employed to analyse intact proteins and retrieve primary sequence information. To our knowledge, top-down proteomics methods with Q-ToF have only been evaluated using samples of relatively low complexity. Furthermore, the in-source CID (IS-CID) capability of Q-ToF instruments has been under-utilised. This study aimed at optimising top-down sequencing of intact milk proteins to achieve the greatest sequence coverage possible from samples of increasing complexity, assessed using nine known proteins. Eleven MS/MS methods varying in their IS-CID and conventional CID parameters were tested on individual and mixed protein standards as well as raw milk samples. Top-down sequencing results from the nine most abundant proteoforms of caseins, alpha-lactalbumin and beta-lactoglubulins were compared. Nine MS/MS methods achieved more than $70 \%$ sequence coverage overall to distinguish between allelic proteoforms, varying only by one or two amino acids. The optimal methods utilised IS-CID at low energy. This experiment demonstrates the utility of Q-ToF systems for top-down proteomics and that IS-CID could be more frequently employed.
\end{abstract}

Keywords: top-down proteomics; HPLC-ESI-Q-TOF MS; cow's milk; whey proteins and caseins; tandem MS

\section{Introduction}

Top-down proteomics, a term invented by Kelleher and colleagues 20 years ago [1], describes the analysis of intact proteins, either in their native form or more often in a denatured state, which allows for a characterisation of proteoforms as comprehensively as possible. Coined in 2014, the term proteoform "designates all of the different molecular forms in which the protein product of a single gene can be found, encompassing all forms of genetic variation, alternative splicing of RNA transcripts, and post-translational modifications (PTMs)" [2]. The analysis of intact proteins is now always performed using mass spectrometry (MS) and technical progresses in top-down proteomics are tightly linked to improvements made on mass analysers. The most important technological advance was the coupling of the soft ionisation technique, electrospray ionisation (ESI), to a mass spectrometer and the production of gas phase ions from large molecules [3], which led to its first application to 
intact proteins [4,5]. ESI generates multiply-charged protein ions of low $m / z$, thereby allowing the analysis of very large molecules, even on an MS platform with a limited mass range. By applying a deconvolution algorithm [6], the resulting complex multi-peak spectrum can be converted into a single peak corresponding to the true molecular weight of the molecule. Further developments in intact protein analysis quickly followed with the introduction of complex multistage mass analysers of high resolution such as linear ion trap (LIT), quadrupole time-of-flight (Q-ToF), Fourier transform (FT) ion cyclotron resonance, and orbitrap instruments.

Today, various methods of ion fragmentation are available, such as collision-induced dissociation (CID, also called collisionally activated dissociation CAD), in-source CID (IS-CID, also known as up-front CID, cone-voltage CID, and nozzle-skimmer dissociation), higher-energy collisional dissociation (HCD), electron capture dissociation (ECD), and electron transfer dissociation (ETD) (for review [7]). Different types of fragmentation modes yield different information about the structure and composition of the analyte. Tandem mass spectrometers that carry out CID to generate product ions from precursor ions have proven extremely useful for the identification and characterisation of proteins from a complex mixture. CID was first described by McLafferty and Bryce in 1967 [8] and Jennings in 1968 [9]. Parent ions collide with neutral gas atoms or molecules (typically helium, nitrogen or argon), which result in the formation of b-and y-type ions. The efficacy of a CID experiment will depend on the relative translational energy of the ion and target, the nature of the target, the number of collisions that is likely to take place, and the $m / z$ window of the instrument. Initial top-down sequencing experiments exploited low-energy CID methods to induce protein fragmentation. However, for large molecules, CID does not produce a fragmentation pattern comprehensive enough to fully characterise proteins, but rather produces enough fragments or sequence tags to identify the protein. Additionally, if the protein contains PTMs, low-energy CID most likely will not be sufficient to localise the modified site or the PTM may be the preferred site of cleavage [10,11]. IS-CID is a proven albeit seldom utilised fragmentation mode allowing single-stage instrumentation, such as single quadrupole (Q) or ToF mass analysers, to produce spectra similar to those obtained with far more expensive hybrid instruments. In this method, invented by Katta and colleagues in 1991 [12], CID is carried out within the ion source in the high-pressure region between the capillary exit and the skimmer entrance to the $\mathrm{Q}$ mass spectrometer. Because there is no prior selection of the precursor ion, this does not qualify as a bona fide MS/MS experiment. By manipulating lens voltages that channel ions from the source to the mass analyser, relatively low-energy ions formed in the atmospheric pressure region of the source collide with residual background gas, usually nitrogen, in the transition region. As a result, excited ions can undergo unimolecular decomposition to produce fragment ions. As for CID, only b- and y-type ions are observed. Tandem spectra from protonated peptides produced by IS-CID or low-energy CID are comparable [13,14]. In-source dissociation has also been demonstrated for whole protein ions [15]. The time scale for IS-CID is on the order of a few hundred microseconds to a few milliseconds, which is much faster than that of CID. IS-CID fragmentation can be reproducible provided ion source parameters, such as temperature, pressure, voltage, and sample purity are tightly controlled [16].

Kelleher in 2004 [17] rigorously defines top-down proteomics as a multistep process whereby the molecular weights (MWs) of intact proteoforms are accurately measured using a high-resolution mass analyser in combination with a direct fragmentation of the protein ions using tandem MS. Top-down sequencing of intact proteins were initially performed using triple quadrupole (QQQ) instruments and IS-CID [5]. In 1990, Loo and colleagues characterised a 14-kDa bovine ribonuclease A in its native and reduced forms using CID [18]. Soon after, higher mass resolution was achieved by applying FT-MS to equine cytochrome c, porcine albumin, thioredoxin and ubiquitin [19], myoglobin [20], and carbonic anhydrase [15]. A hybrid Q-ToF instrument, also called Q-ToF [21], was invented in 1996 by Morris and colleagues [22]. Q-ToF mass spectrometers combine the quadrupole one (Q1) in which ion precursors are funnelled through and selected, the quadrupole collision cell (q) of a QQQ in which selected precursor ions are fragmented usually through CID and more recently ETD, and a reflector ToF 
detector in which the fragment ions are mass analysed. Spectra obtained in both full-scan (MS1) and MS/MS (MS2) modes exhibit clean spectra due to orthogonal pulsing into the ToF section, high mass accuracy along with stable isotopic resolution across the $m / z$ range, and high fidelity meaning accurate isotopic ratios. This permits the determination of charge states and unambiguous assignment of the mono-isotopic signal of the intact molecules. This leads to accurate prediction of AA sequence and successful identification of peptides and proteins of medium MW via database searches by tightening the search parameters and augmenting the confidence in the results. Q-ToF instruments perform well for quantitative analyses [23] and for the identification of PTMs [24]. Q-ToF were demonstrated to outperform QQQ mass spectrometers owing to both their enhanced sensitivity and resolution in the region of one to two orders of magnitude [22]. This initial observation was further validated on phosphopeptides; the higher resolving power of the Q-ToF improved the selectivity and sensitivity of parent ions, thus minimising interference from other product ions and maximising sequencing results [25].

The first use of LC-ESI-Q-ToF MS in top-down sequencing of intact proteins was reported by Nemeth-Cawley and Rouse in 2002 [26], who used CID to fragment ion precursors from eight known protein standards, ranging from 5 to $66 \mathrm{kDa}$. Peptide sequence tags thus obtained led to the unambiguous identification of the analysed proteins, along with the characterisation of disulphide bonds and glycosylation. While their method was successful, the authors noted some limitations: first, it could not yet be applied to complex protein mixtures without prior separation; secondly, at a low concentration, the signal-to-noise ratio was affected, thus necessitating longer MS/MS acquisition times. Based on their experience, the following year, the same group applied their validated method to characterise a recombinant immunoglobulin gamma-1 (IgG-1) fusion protein [27]. The top-down strategy allowed them to demonstrate that the recombinant protein was expressed as a full-length form as well as N- and C-processed truncated proteoforms [27]. In 2004, Ginter and colleagues exploited both IS-CID and traditional CID mode of a Q-ToF mass spectrometer, thereby achieving pseudo-MS3 levels, to top-down sequence seven known proteins spanning from 11 to $66 \mathrm{kDa}$ [28]. Individual sequence tags of 10 to 26 AAs were retrieved from both N- and C-termini, and unambiguous identification of protein standards was achieved. IS-CID on its own was used to identify various purified recombinant proteins using ESI-Q-ToF MS [29]; fragmentation efficiency depended on cone voltage and y-ions formed predominantly by cleavage on the C-terminal side of nonpolar residues. An unknown 4.9 kDa recombinant peptide was completely sequenced using LC-ESI-Q-ToF MS/MS of 10,000 resolution and traditional CID fragmentation; complete $y$ - and b-type ion series were obtained and the formation of beta-mercaptoethanol adducts was reported [30]. In 2009 Armirotti and colleagues reported 90-100\% sequence coverage of horse myoglobin (17 kDa) and bovine carbonic anhydrase II (30 kDa) using LC-ESI-Q-ToF MS/MS and CID fragmentation mode [31]. They could also identify an unknown protein as superoxide dismutase $(16 \mathrm{kDa})$ and locate one acetylation site. ESI CID Q-TOF MS/MS was used to identify eight intact antimicrobial peptides from Asian frog skin and locate disulphide bridges [32]. ESI-Q-ToF technology has also been employed to investigate the precise stoichiometry of protein assemblies, the interactions between subunits and the position of subunits within the complex [33]. 
In our laboratory, we host a maXis HD UHR-Q-ToF (60,000 resolution) with an ESI source on-line with a UHPLC 1290 Infinity Binary LC system (Agilent, Mulgrave, VIC, Australia), which we have used to develop a top-down method to analyse cow's milk proteins [23]. This method was further implemented with CID MS/MS analysis and applied to the study of UHT milk shelf life [34]. The present study aimed at optimising MS/MS analysis by testing the effect of some of the parameters pertaining to IS-CID in combination or not with conventional CID. A total of $11 \mathrm{MS} / \mathrm{MS}$ methods were assessed on samples bearing increased complexity, namely individual milk protein standards, mixed protein standards and cow's raw milk samples from Jersey or Holstein breeds. Milk proteins exhibit numerous PTMs, however in this initial study, we focused on optimising protein fragmentation to achieve the greatest sequence coverage possible. To this end, nine of the most abundant proteoforms of milk caseins and whey proteins, including genetic variants, were compared across samples and MS/MS methods whose efficacy was assessed based on protein sequence coverage alone. This necessitated developing processing workflows using the powerful Genedata Expressionist software to thoroughly explore and annotate the MS1 and MS2 files.

\section{Results and Discussion}

\subsection{Sample Complexity}

In this experiment, we wanted to test whether sample complexity would impact the quality of spectral acquisition. To this end, we prepared a set of samples displaying increasing proteome complexity: from simple individual milk protein standards, albeit varying in their purity level from $70 \%$ $(\alpha-C N)$ to $98 \%(\beta-C N)$, to a mixture of these standards, to the highly complex biological matrices raw milk samples that were obtained from two common cow breeds. Figure 1 describes the experimental design of this study.

\subsubsection{UPLC-ESI-Q-ToF MS Can Handle Complex Samples}

While the raw data clearly illustrate increasing complexity with more LC-MS1 peaks detected in the milk samples compared to the individual protein standard samples (Figure 2A), the latter are more complex than first anticipated, particularly the $\beta-\mathrm{CN}$ standard, which is claimed to be $98 \%$ pure.

These standards were analysed using a bottom-up shotgun approach and yielded many protein hits [35]. The $\alpha$-LA standard sample ( $85 \%$ pure) generated by far the simplest LC-MS1 pattern, with clear elution from 14 to $19 \mathrm{~min}$ and distinct $m / z$ peaks of the charged envelope (Figure 2A).

The LC-MS1 pattern of the mixed standard sample is quite similar and as complex as the patterns of milk samples owing to the fact that the individual protein standards are far from pure. Indeed, following protein mass deconvolution and the display of protein accurate masses separated by HPLC (Figure 2B), many deconvoluted peaks appear besides the expected proteins of interest. Such a display confirms that the $\alpha$-LA standard is the least complex sample with only 211 deconvoluted peaks, whereas the other individual standards display between $2194(\alpha-\mathrm{CN})$ and 3372 peaks $(\beta-\mathrm{CN})$.

With 4819 deconvoluted peaks, the mixed standard sample is indeed of intermediate complexity between the individual standards and the raw milk samples, which resolved 6988 and 7554 peaks in Jersey and Holstein cows, respectively. While proteins displayed some degree of co-elution during the HPLC separation, particularly in complex samples, separation along the $m / z$ range could isotopically resolve all proteins of less than $30 \mathrm{kDa}$, thus yielding deconvoluted monoisotopic masses (also shown in [23]). 


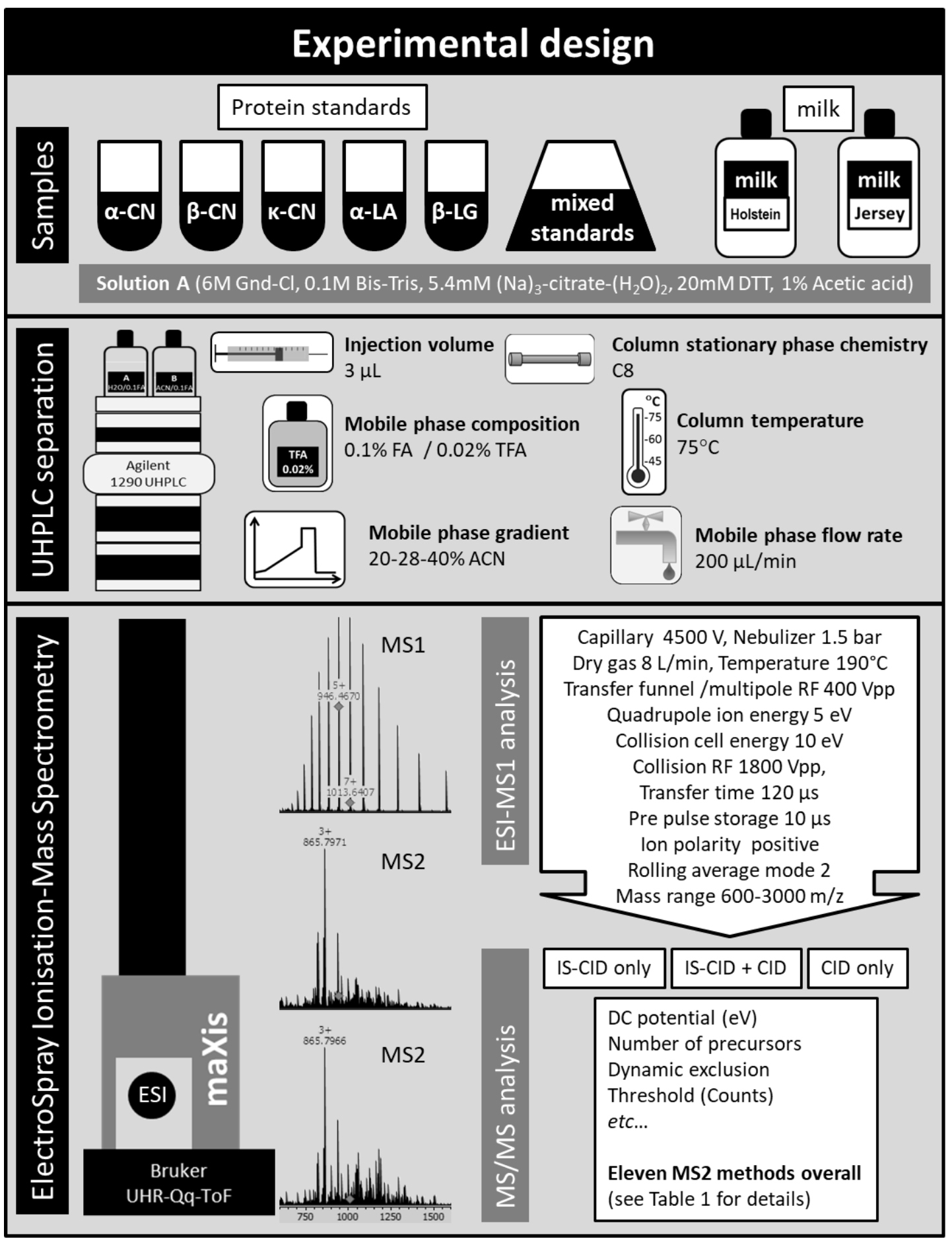

Figure 1. Schematic diagram of the experimental design of the study. 


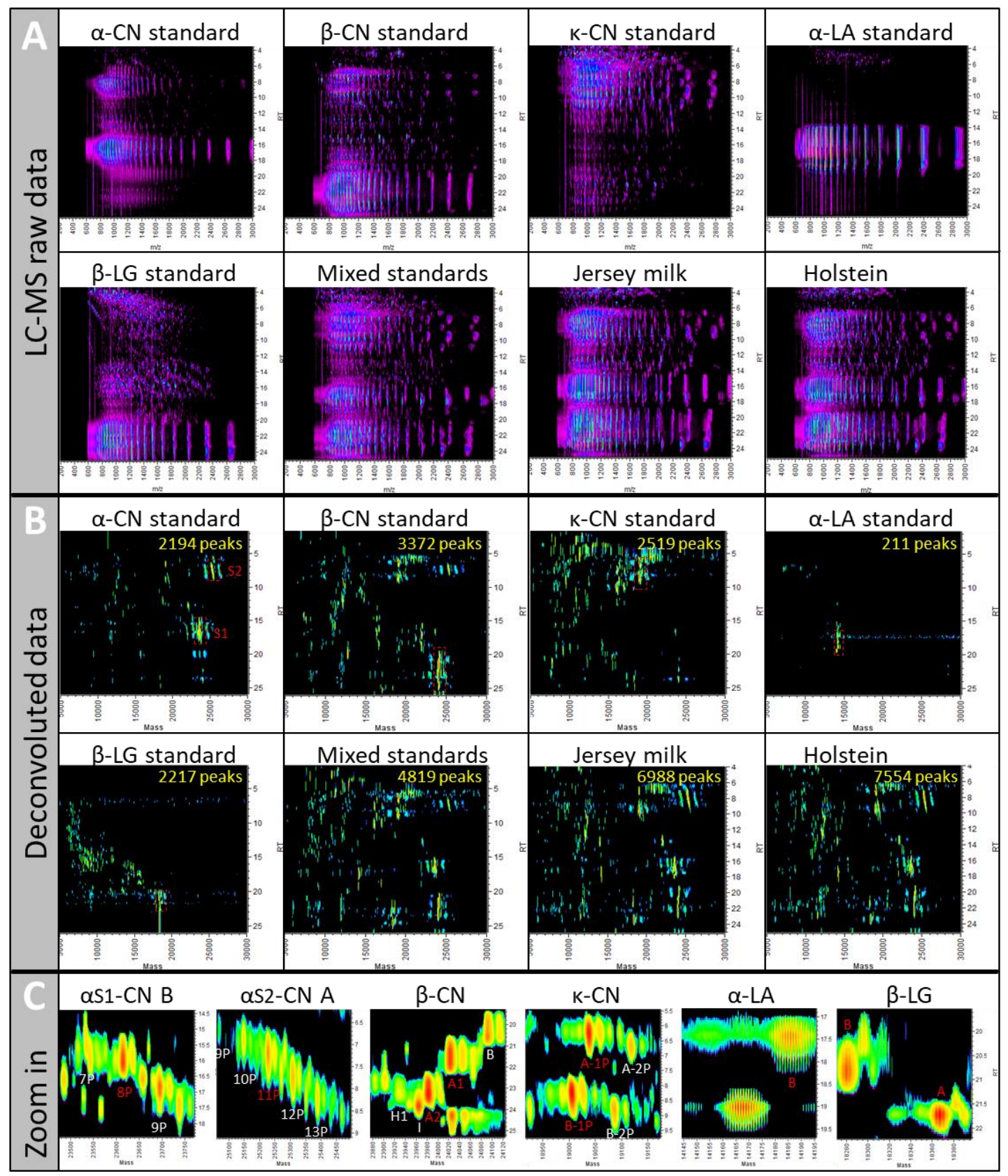

Figure 2. LC-MS maps of the different samples (individual protein standards, mixed protein standards, Jersey milk, and Holstein milk) visualised in Genedata Refiner pre- and post-deconvolution with $\mathrm{m} / \mathrm{z}$ on the $\mathrm{x}$ axis and LC retention time in $\min$ on the $\mathrm{y}$ axis. (A) LC-MS1 unprocessed raw data. (B) Deconvoluted data displaying proteins of interest in the red boxed areas. The total numbers of deconvoluted peaks detected are indicated in yellow. (C) Close-ups of the boxed areas of the individual protein standards; the nine proteoforms highlighted in red are the proteins targeted for method validation. 


\subsubsection{The $11 \mathrm{MS} / \mathrm{MS}$ Methods Are Assessed Using a Reference Set of Known Proteins}

Another aim of our study was to optimise the MS/MS method by modifying key parameters and devise objective tools to compare the different methods. We thought the best comparative approach would be a targeted one relying on a small reference set of known proteins. The proteins of interest are depicted in the boxed areas of Figure 2B which have been zoomed in in Figure 2C. We chose nine prominent proteoforms, some of them displaying allelic variations, in each of the individual standards, namely, $\alpha-\mathrm{CN}$ type $\mathrm{S} 1$ variant B with 8 phosphorylations $\left(\alpha_{\mathrm{S} 1}-\mathrm{CN} \mathrm{B}-8 \mathrm{P}, 23,600.3 \mathrm{Da}\right), \alpha-\mathrm{CN}$ type $\mathrm{S} 2$ variant $A$ with 11 phosphorylations ( $\alpha_{\mathrm{S} 2}-\mathrm{CN}$ A-11P, 25,213.0 Da), $\beta-\mathrm{CN}$ variants A1 and A2 ( $\beta-\mathrm{CN}$ A1 24,008.2 Da, $\beta-C N$ A2 23,968.2 Da) each with five phosphorylations, $\kappa-C N$ variants $A$ and $B$ with 1

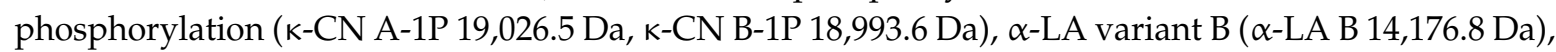
and $\beta$-LG variants A and B ( $\beta$-LG A 18,355.5 Da, $\beta$-LG B 18269.4 Da). We have previously used these proteins to optimise a quantitative LC-MS method [23]. The full primary sequence of these proteins is known, and therefore fragmentation efficiency for top-down sequencing purpose could be assessed. This is presented in the last chapter. While not included in this article, our MS/MS method also allowed us to identify various PTMs of milk most abundant proteins such as lactosylation (+324 Da), oxidation (+16 Da), glycosylation $([\mathrm{Hex}(1) \mathrm{HexNAc}(1) \mathrm{NeuAc}(2)]+947 \mathrm{Da})$, and degradation products, as reported in [34].

\subsection{Effect of IS-CID and CID on Their Own or Combined on Spectral Data}

In a recent study [34], we have used conventional CID (Method 2 in the present study) to identify the degradation products of milk's most abundant proteins occurring following UHT treatment and storage on the shelf. While this top-down sequencing method proved successful for the identification of small proteins and degradation products, we wanted to optimise it for the analysis of intact milk proteins. Our Q-ToF mass spectrometer allows us to perform both IS-CID and CID in a single experiment. The benefits of both have been stated in the introduction of this manuscript. In the present study, we have only slightly varied the parameters controlling CID fragmentation, choosing to operate it in a fully automated manner. We have mostly fine-tuned the parameters pertaining to IS-CID. We thus tested different energy values for IS-CID MS (ion funnel 1 exit) and IS-CID MS/MS (ion funnel 2 entrance) parameters. A total of 11 MS/MS methods were thus devised that employed either CID or IS-CID on their own or combined (Table 1).

In summary, Methods 2 and 3 employed CID only, Methods 4 and 12 employed IS-CID only, and Methods 5-11 employed IS-CID within the ESI source/ion transfer region, followed by CID within the collision cell. 
Table 1. Parameters applied on the Q-TOF mass spectrometer for the 12 MS methods.

\begin{tabular}{|c|c|c|c|c|c|c|c|c|c|c|c|c|c|c|}
\hline Mode & Step & Parameter & $\begin{array}{c}\text { Method } \\
1\end{array}$ & $\begin{array}{c}\text { Method } \\
2\end{array}$ & $\begin{array}{c}\text { Method } \\
3\end{array}$ & $\begin{array}{c}\text { Method } \\
4\end{array}$ & $\begin{array}{c}\text { Method } \\
5\end{array}$ & $\begin{array}{c}\text { Method } \\
6\end{array}$ & $\begin{array}{c}\text { Method } \\
7\end{array}$ & $\begin{array}{c}\text { Method } \\
8\end{array}$ & $\begin{array}{l}\text { Method } \\
9\end{array}$ & $\begin{array}{c}\text { Method } \\
10\end{array}$ & $\begin{array}{c}\text { Method } \\
11\end{array}$ & $\begin{array}{c}\text { Method } \\
12\end{array}$ \\
\hline MS & Source & End plate offset & $500 \mathrm{~V}$ & $500 \mathrm{~V}$ & $500 \mathrm{~V}$ & $500 \mathrm{~V}$ & $500 \mathrm{~V}$ & $500 \mathrm{~V}$ & $500 \mathrm{~V}$ & $500 \mathrm{~V}$ & $500 \mathrm{~V}$ & $500 \mathrm{~V}$ & $500 \mathrm{~V}$ & $500 \mathrm{~V}$ \\
\hline MS & Source & Capillary & $4500 \mathrm{~V}$ & $4500 \mathrm{~V}$ & $4500 \mathrm{~V}$ & $4500 \mathrm{~V}$ & $4500 \mathrm{~V}$ & $4500 \mathrm{~V}$ & $4500 \mathrm{~V}$ & $4500 \mathrm{~V}$ & $4500 \mathrm{~V}$ & $4500 \mathrm{~V}$ & $4500 \mathrm{~V}$ & $4500 \mathrm{~V}$ \\
\hline MS & Source & Nebuliser & $1.5 \mathrm{bar}$ & $1.5 \mathrm{bar}$ & $1.5 \mathrm{bar}$ & $1.5 \mathrm{bar}$ & $1.5 \mathrm{bar}$ & $1.5 \mathrm{bar}$ & $1.5 \mathrm{bar}$ & $1.5 \mathrm{bar}$ & $1.5 \mathrm{bar}$ & $1.5 \mathrm{bar}$ & $1.5 \mathrm{bar}$ & $1.5 \mathrm{bar}$ \\
\hline MS & Source & Dry gas & $8 \mathrm{~L} / \mathrm{min}$ & $8 \mathrm{~L} / \min$ & $8 \mathrm{~L} / \mathrm{min}$ & $8 \mathrm{~L} / \mathrm{min}$ & $8 \mathrm{~L} / \min$ & $8 \mathrm{~L} / \mathrm{min}$ & $8 \mathrm{~L} / \mathrm{min}$ & $8 \mathrm{~L} / \mathrm{min}$ & $8 \mathrm{~L} / \mathrm{min}$ & $8 \mathrm{~L} / \mathrm{min}$ & $8 \mathrm{~L} / \mathrm{min}$ & $8 \mathrm{~L} / \mathrm{min}$ \\
\hline MS & Source & Temperature & $190^{\circ} \mathrm{C}$ & $190^{\circ} \mathrm{C}$ & $190^{\circ} \mathrm{C}$ & $190^{\circ} \mathrm{C}$ & $190^{\circ} \mathrm{C}$ & $190^{\circ} \mathrm{C}$ & $190^{\circ} \mathrm{C}$ & $190^{\circ} \mathrm{C}$ & $190^{\circ} \mathrm{C}$ & $190^{\circ} \mathrm{C}$ & $190^{\circ} \mathrm{C}$ & $190^{\circ} \mathrm{C}$ \\
\hline MS & Source & Waste (min) & $0-2.5$ & $0-2.5$ & $0-2.5$ & $0-2.5$ & $0-2.5$ & $0-2.5$ & $0-2.5$ & $0-2.5$ & $0-2.5$ & $0-2.5$ & $0-2.5$ & $0-2.5$ \\
\hline MS & Source & Source (min) & $2.5-40$ & $2.5-40$ & $2.5-40$ & $2.5-40$ & $2.5-40$ & $2.5-40$ & $2.5-40$ & $2.5-40$ & $2.5-40$ & $2.5-40$ & $2.5-40$ & $2.5-40$ \\
\hline MS & Transfer & Funnel RF & $400 \mathrm{Vpp}$ & $400 \mathrm{Vpp}$ & $400 \mathrm{Vpp}$ & $400 \mathrm{Vpp}$ & $400 \mathrm{Vpp}$ & $400 \mathrm{Vpp}$ & $400 \mathrm{Vpp}$ & $400 \mathrm{Vpp}$ & $400 \mathrm{Vpp}$ & $400 \mathrm{Vpp}$ & $400 \mathrm{Vpp}$ & $400 \mathrm{Vpp}$ \\
\hline MS & Transfer & IS-CID energy & $0 \mathrm{eV}$ & $0 \mathrm{eV}$ & $0 \mathrm{eV}$ & $3 \mathrm{eV}$ & $30 \mathrm{eV}$ & $50 \mathrm{eV}$ & $70 \mathrm{eV}$ & $5 \mathrm{eV}$ & $0 \mathrm{eV}$ & $3 \mathrm{eV}$ & $0 \mathrm{eV}$ & $10 \mathrm{eV}$ \\
\hline MS & Transfer & $\begin{array}{c}\text { Transfer multipole } \\
\text { RF }\end{array}$ & $400 \mathrm{Vpp}$ & $400 \mathrm{Vpp}$ & $400 \mathrm{Vpp}$ & $400 \mathrm{Vpp}$ & 400 Vpp & $400 \mathrm{Vpp}$ & 400 Vpp & $400 \mathrm{Vpp}$ & $400 \mathrm{Vpp}$ & $400 \mathrm{Vpp}$ & $400 \mathrm{Vpp}$ & $400 \mathrm{Vpp}$ \\
\hline MS & Quadrupole & Ion energy & $5 \mathrm{eV}$ & $5 \mathrm{eV}$ & $5 \mathrm{eV}$ & $5 \mathrm{eV}$ & $5 \mathrm{eV}$ & $5 \mathrm{eV}$ & $5 \mathrm{eV}$ & $5 \mathrm{eV}$ & $5 \mathrm{eV}$ & $5 \mathrm{eV}$ & $5 \mathrm{eV}$ & $5 \mathrm{eV}$ \\
\hline MS & Quadrupole & Low mass & $300 \mathrm{~m} / \mathrm{z}$ & $300 \mathrm{~m} / \mathrm{z}$ & $300 \mathrm{~m} / \mathrm{z}$ & $300 \mathrm{~m} / \mathrm{z}$ & $300 \mathrm{~m} / \mathrm{z}$ & $300 \mathrm{~m} / \mathrm{z}$ & $300 \mathrm{~m} / \mathrm{z}$ & $300 \mathrm{~m} / \mathrm{z}$ & $300 \mathrm{~m} / \mathrm{z}$ & $300 \mathrm{~m} / \mathrm{z}$ & $300 \mathrm{~m} / \mathrm{z}$ & $300 \mathrm{~m} / \mathrm{z}$ \\
\hline MS & Collision cell & Collision energy & $10 \mathrm{eV}$ & $10 \mathrm{eV}$ & $10 \mathrm{eV}$ & $10 \mathrm{eV}$ & $10 \mathrm{eV}$ & $10 \mathrm{eV}$ & $10 \mathrm{eV}$ & $10 \mathrm{eV}$ & $10 \mathrm{eV}$ & $10 \mathrm{eV}$ & $10 \mathrm{eV}$ & $10 \mathrm{eV}$ \\
\hline MS & Collision cell & Collision RF (Vpp) & 1800 & 1800 & 1800 & 1800 & 1800 & 1800 & 1800 & 1800 & 1800 & 1800 & 1800 & 1800 \\
\hline MS & Collision cell & Transfer time & $120 \mu \mathrm{s}$ & $120 \mu \mathrm{s}$ & $120 \mu \mathrm{s}$ & $120 \mu \mathrm{s}$ & $120 \mu \mathrm{s}$ & $120 \mu \mathrm{s}$ & $120 \mu \mathrm{s}$ & $120 \mu \mathrm{s}$ & $120 \mu \mathrm{s}$ & $150 \mu \mathrm{s}$ & $120 \mu \mathrm{s}$ & $120 \mu \mathrm{s}$ \\
\hline MS & Collision cell & Pre-pulse storage & $10 \mu \mathrm{s}$ & $10 \mu \mathrm{s}$ & $10 \mu \mathrm{s}$ & $10 \mu \mathrm{s}$ & $10 \mu \mathrm{s}$ & $10 \mu \mathrm{s}$ & $10 \mu \mathrm{s}$ & $10 \mu \mathrm{s}$ & $10 \mu \mathrm{s}$ & $15 \mu \mathrm{s}$ & $10 \mu \mathrm{s}$ & $10 \mu \mathrm{s}$ \\
\hline MS & MS1 & Ion polarity & positive & positive & positive & positive & Positive & Positive & positive & positive & positive & positive & positive & positive \\
\hline MS & MS1 & Mass range $(\mathrm{m} / \mathrm{z})$ & $600-3000$ & $600-3000$ & $600-3000$ & $200-2000$ & $200-2000$ & $200-2000$ & $200-2000$ & $200-2000$ & $200-2000$ & $200-2000$ & $200-2000$ & $200-2000$ \\
\hline MS & MS1 & Summation & 15,000 & 15,000 & 15,000 & 18,321 & 18,321 & 18,321 & 18,321 & 18,321 & 18,321 & 18,321 & 18,321 & 18,321 \\
\hline MS & MS1 & $\begin{array}{l}\text { Rolling average } \\
\text { mode }\end{array}$ & 2 & 2 & 2 & 2 & 2 & 2 & 2 & 2 & 2 & 2 & 2 & 2 \\
\hline in source & IS-CID & IS-CID & NO & NO & NO & YES & YES & YES & YES & YES & YES & YES & YES & YES \\
\hline in source & IS-CID & $\begin{array}{c}\text { IS-CID MS } \\
\text { (funnel } 1 \text { exit) }\end{array}$ & & & & $3 \mathrm{eV}$ & $30 \mathrm{eV}$ & $50 \mathrm{eV}$ & $70 \mathrm{eV}$ & $5 \mathrm{eV}$ & $3 \mathrm{eV}$ & $3 \mathrm{eV}$ & $3 \mathrm{eV}$ & $10 \mathrm{eV}$ \\
\hline in source & IS-CID & $\begin{array}{l}\text { IS-CID MS/MS } \\
\text { (funnel } 2 \text { entry) }\end{array}$ & & & & $15 \mathrm{eV}$ & $10 \mathrm{eV}$ & $10 \mathrm{eV}$ & $10 \mathrm{eV}$ & $20 \mathrm{eV}$ & $10 \mathrm{eV}$ & $30 \mathrm{eV}$ & $10 \mathrm{eV}$ & $15 \mathrm{eV}$ \\
\hline in source & IS-CID & $\begin{array}{l}\text { Acquisition time } \\
\text { factor }\end{array}$ & & & & 1 & 1 & 1 & 1 & 1 & 1 & 1 & 1 & 1 \\
\hline MS/MS & MS2 & $\begin{array}{l}\text { Auto MS/MS } \\
\text { mode }\end{array}$ & OFF & ON & $\mathrm{ON}$ & OFF & ON & ON & ON & ON & $\mathrm{ON}$ & $\mathrm{ON}$ & $\mathrm{ON}$ & OFF \\
\hline MS/MS & CID & Acquisition & $\mathrm{NO}$ & YES & YES & $\mathrm{NO}$ & YES & YES & YES & YES & YES & YES & YES & NO \\
\hline MS/MS & CID & $\begin{array}{c}\text { MS/MS low } \\
\text { (per } 1000 \text { sum.) }\end{array}$ & & $10^{4} \mathrm{cts}$ & $10^{4} \mathrm{cts}$ & & $10^{4} \mathrm{cts}$ & $10^{4} \mathrm{cts}$ & $10^{4} \mathrm{cts}$ & $10^{4} \mathrm{cts}$ & $10^{4} \mathrm{cts}$ & $10^{4} \mathrm{cts}$ & $10^{4} \mathrm{cts}$ & \\
\hline MS/MS & CID & $\begin{array}{l}\text { MS/MS high } \\
\text { (per } 1000 \text { sum.) }\end{array}$ & & $10^{6} \mathrm{cts}$ & $10^{6} \mathrm{cts}$ & & $10^{6} \mathrm{cts}$ & $10^{6} \mathrm{cts}$ & $10^{6} \mathrm{cts}$ & $10^{6} \mathrm{cts}$ & $10^{6} \mathrm{cts}$ & $10^{6} \mathrm{cts}$ & $10^{6} \mathrm{cts}$ & \\
\hline MS/MS & MS2 & $\begin{array}{l}\text { Preference } \\
\text { activated }\end{array}$ & $\mathrm{NO}$ & $\mathrm{NO}$ & $\mathrm{NO}$ & $\mathrm{NO}$ & $\mathrm{NO}$ & $\mathrm{NO}$ & $\mathrm{NO}$ & $\mathrm{NO}$ & YES & $\mathrm{NO}$ & $\mathrm{NO}$ & NO \\
\hline
\end{tabular}


Table 1. Cont.

\begin{tabular}{|c|c|c|c|c|c|c|c|c|c|c|c|c|c|c|}
\hline Mode & Step & Parameter & $\begin{array}{c}\text { Method } \\
1\end{array}$ & $\begin{array}{c}\text { Method } \\
2\end{array}$ & $\begin{array}{c}\text { Method } \\
3\end{array}$ & $\begin{array}{c}\text { Method } \\
\quad 4\end{array}$ & $\begin{array}{c}\text { Method } \\
5\end{array}$ & $\begin{array}{c}\text { Method } \\
6\end{array}$ & $\begin{array}{c}\text { Method } \\
7\end{array}$ & $\begin{array}{c}\text { Method } \\
8\end{array}$ & $\begin{array}{l}\text { Method } \\
9\end{array}$ & $\begin{array}{c}\text { Method } \\
10\end{array}$ & $\begin{array}{c}\text { Method } \\
11\end{array}$ & $\begin{array}{l}\text { Method } \\
12\end{array}$ \\
\hline MS/MS & MS2 & $\begin{array}{l}\text { Charge state } \\
\text { preferred range }\end{array}$ & & & & & & & & & $7-15$ & & & \\
\hline MS/MS & MS2 & Exclude singly & & & & & & & & & YES & & & \\
\hline MS/MS & Precursor ions & Exclude $(m / z)$ & & $100-600$ & $100-600$ & & $100-600$ & $100-600$ & $100-600$ & $100-600$ & $100-600$ & $100-600$ & $100-600$ & \\
\hline MS/MS & Precursor ions & No. of precursors & & 2 & 2 & & 2 & 2 & 2 & 3 & 4 & 4 & 4 & \\
\hline MS/MS & Threshold & $\begin{array}{c}\text { Absolute } \\
\text { (per } 1000 \text { sum.) }\end{array}$ & & $1153 \mathrm{cts}$ & $170 \mathrm{cts}$ & & $10 \mathrm{cts}$ & $10 \mathrm{cts}$ & $10 \mathrm{cts}$ & $10 \mathrm{cts}$ & $10 \mathrm{cts}$ & $10 \mathrm{cts}$ & $10 \mathrm{cts}$ & \\
\hline MS/MS & Threshold & Absolute & & 17,302 cts & $2550 \mathrm{cts}$ & & $183 \mathrm{cts}$ & $183 \mathrm{cts}$ & $183 \mathrm{cts}$ & $183 \mathrm{cts}$ & $183 \mathrm{cts}$ & $183 \mathrm{cts}$ & $183 \mathrm{cts}$ & \\
\hline MS/MS & Active exclusion & $\mathrm{ON} / \mathrm{OFF}$ & & ON & ON & & ON & ON & ON & ON & ON & ON & ON & \\
\hline MS/MS & Active exclusion & Exclude after & & 3 spectra & 3 spectra & & 1 spectra & 1 spectra & 1 spectra & 1 spectra & 1 spectra & 1 spectra & 1 spectra & \\
\hline MS/MS & Active exclusion & Release after & & $5 \mathrm{~min}$ & $5 \mathrm{~min}$ & & $5 \mathrm{~min}$ & $5 \mathrm{~min}$ & $5 \mathrm{~min}$ & $5 \mathrm{~min}$ & $5 \mathrm{~min}$ & $5 \mathrm{~min}$ & $5 \min$ & \\
\hline
\end{tabular}


Figure 3 displays the LC-MS patterns for each of the 12 methods using the mixed protein standards sample.
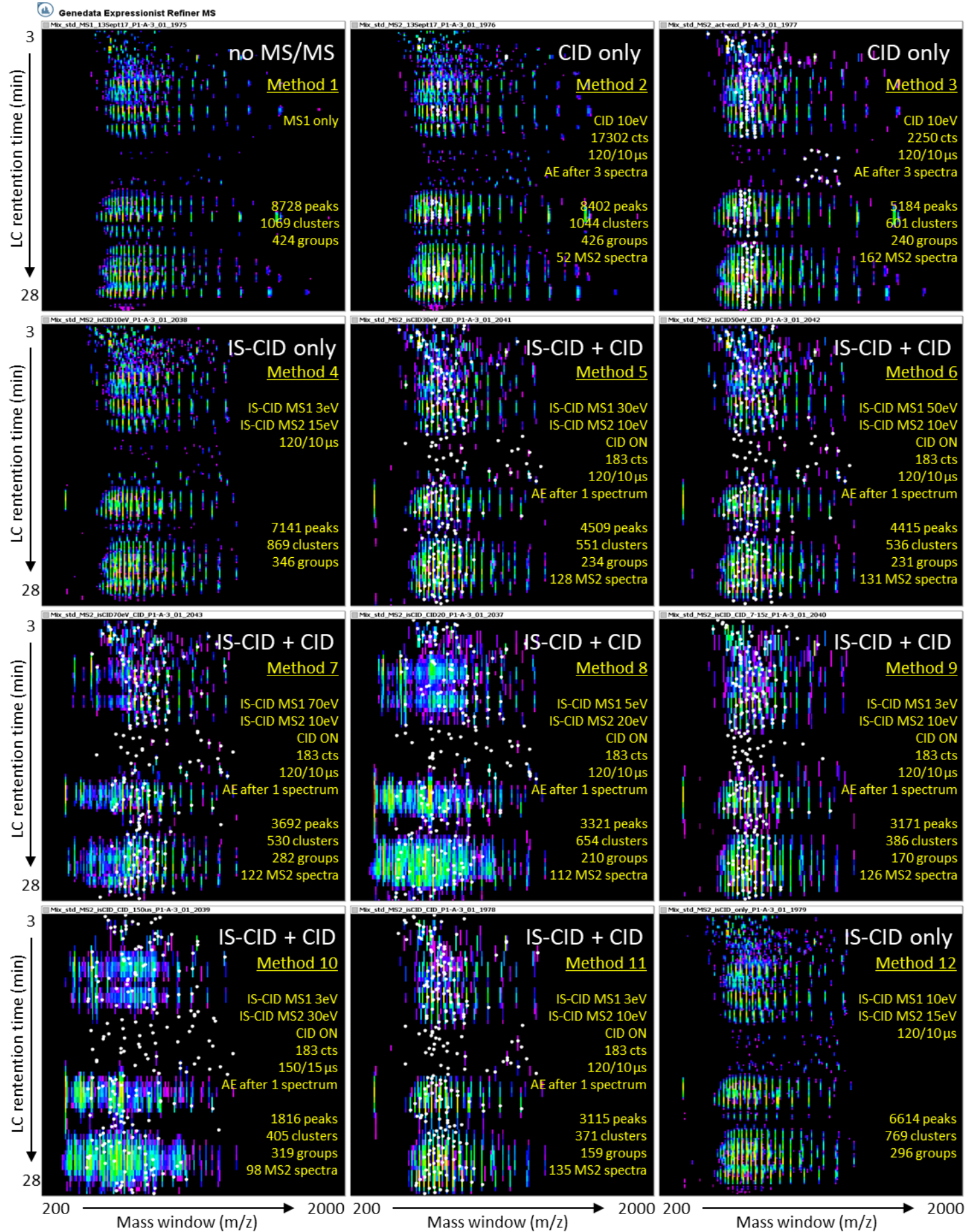

Figure 3. LC-MS maps of the mixed protein standards sample visualised in Genedata Refiner with the scanned mass range (200-2000 $\mathrm{m} / \mathrm{z}$ ) on the $\mathrm{x}$ axis and LC retention time (3-28 $\mathrm{min}$ ) on the y axis across all 12 methods following data processing. White dots represent true MS/MS (MS2) events using conventional CID fragmentation. AE, active exclusion. 
Table 2 compares all 12 methods based on LC-MS and LC-MS/MS observations

Table 2. Number of spectral peaks, clusters, groups and MS/MS spectra found in the mixed standard sample across all 12 methods using Genedata Refiner workflow.

\begin{tabular}{|c|c|c|c|c|c|c|c|c|c|c|c|c|}
\hline Method & 1 & 2 & 3 & 4 & 5 & 6 & 7 & 8 & 9 & 10 & 11 & 12 \\
\hline $\begin{array}{l}\text { LC separation } \\
\text { (min) }\end{array}$ & 22.5 & 22.5 & 22.5 & 22.5 & 22.5 & 22.5 & 22.5 & 22.5 & 22.5 & 22.5 & 22.5 & 22.5 \\
\hline $\begin{array}{c}\text { LC separation } \\
\text { (s) }\end{array}$ & 1350 & 1350 & 1350 & 1350 & 1350 & 1350 & 1350 & 1350 & 1350 & 1350 & 1350 & 1350 \\
\hline Duty cycle (s) & 2.7 & 13.7 & 13.7 & 5.6 & 13.7 & 13.7 & 13.8 & 19.1 & 24.6 & 24.6 & 24.6 & 5.5 \\
\hline $\begin{array}{l}\text { No. of full MS1 } \\
\text { scans }\end{array}$ & 493 & 99 & 99 & 243 & 99 & 99 & 98 & 71 & 55 & 55 & 55 & 247 \\
\hline $\begin{array}{l}\text { (a) Theor. no. of } \\
\text { MS2 events }\end{array}$ & 0 & 197 & 198 & 0 & 197 & 198 & 196 & 212 & 219 & 219 & 220 & 0 \\
\hline $\begin{array}{l}\text { (b) MS1 peaks } \\
(300-3000 \mathrm{~m} / \mathrm{z})\end{array}$ & 8728 & 8402 & 5184 & 7141 & 4509 & 4415 & 3692 & 3321 & 3171 & 1816 & 3115 & 6614 \\
\hline $\begin{array}{l}\text { (c) MS1 peaks } \\
(700-3000 \mathrm{~m} / \mathrm{z})\end{array}$ & 8711 & 8387 & 5184 & 7124 & 4503 & 4415 & 3599 & 3040 & 3169 & 1628 & 3113 & 6599 \\
\hline $\begin{array}{l}\text { (d) MS1 peaks } \\
(300-700 \mathrm{~m} / \mathrm{z})\end{array}$ & 17 & 15 & 0 & 17 & 6 & 0 & 93 & 281 & 2 & 188 & 2 & 15 \\
\hline $\begin{array}{l}\text { (e) Observed No. } \\
\text { of MS2 spectra }\end{array}$ & 0 & 56 & 170 & 0 & 196 & 196 & 196 & 210 & 218 & 218 & 218 & 0 \\
\hline (e) $/(a)(\%)$ & & 28 & 86 & & 99 & 99 & 100 & 99 & 99 & 99 & 99 & \\
\hline (e)/(b) (\%) & & 0.7 & 3.3 & & 4.3 & 4.4 & 5.3 & 6.3 & 6.9 & 12.0 & 7.0 & \\
\hline MS2 $m / z$ range & & $730-1636$ & 644-1854 & & 643-1955 & 703-1955 & 657-1966 & 609-1913 & $607-1774$ & 609-1966 & 634-1928 & \\
\hline $600-700 \mathrm{~m} / \mathrm{z}$ & & & 1 & & 3 & & 2 & 18 & 3 & 20 & 4 & \\
\hline $700-800 \mathrm{~m} / \mathrm{z}$ & & 2 & 2 & & 5 & 5 & 5 & 14 & 4 & 19 & 8 & \\
\hline $800-900 \mathrm{~m} / \mathrm{z}$ & & 4 & 12 & & 20 & 20 & 15 & 22 & 26 & 27 & 29 & \\
\hline $900-1000 \mathrm{~m} / \mathrm{z}$ & & 20 & 53 & & 45 & 44 & 21 & 34 & 56 & 36 & 54 & \\
\hline $1000-1100 \mathrm{~m} / \mathrm{z}$ & & 28 & 77 & & 53 & 51 & 47 & 23 & 63 & 24 & 57 & \\
\hline $1100-1200 \mathrm{~m} / \mathrm{z}$ & & & 5 & & 29 & 26 & 35 & 24 & 30 & 18 & 23 & \\
\hline $1200-1300 \mathrm{~m} / \mathrm{z}$ & & & 2 & & 19 & 21 & 26 & 26 & 20 & 29 & 18 & \\
\hline $1300-1400 \mathrm{~m} / \mathrm{z}$ & & & 2 & & 3 & 7 & 17 & 14 & 8 & 7 & 2 & \\
\hline
\end{tabular}

\subsubsection{Precursor Intensity Threshold Is a Key Parameter for CID Fragmentation}

Methods 2 and 3 (CID only) only differed in the intensity threshold for precursor selection, being almost 7 times higher in Method 2 (2550 counts) relative to Method 3 (17,302 counts) (Table 1). This allows precursor ions of low signal intensity to undergo CID fragmentation. As expected, this did not affect the LC-MS1 data; however, it did result in many more MS/MS events when Method 3 was used. If we consider the mixed standard sample as an example, Method 2 resulted in 8402 ions with only 56 (0.7\%) MS2 spectra, whereas Method 3 yielded 5184 peaks and 170 (3.3\%) MS2 spectra (Table 2). Consequently, the top-down sequencing efficiency varied between Methods 2 and 3, as will be discussed in the following section.

Charge state preference aside, in this study we did not attempt to optimise CID conditions and took full advantage of the automatic MS/MS mode available on our instrument with default parameters as it proved efficient [34]. Using CID on its own, Nemeth-Cawley and colleagues successfully top-down sequenced unknown intact proteins purified by affinity and size-exclusion chromatography from mammalian cells using an ESI-Q-ToF MS/MS strategy [27].

\subsubsection{Ion Funnel 1 Energy Has Little Impact on IS-CID Fragmentation}

Methods 4 and 12 (IS-CID only) differed only in the energy applied to the ion funnel 1 exit during the IS-CID MS1 step. Method 4 applied $3 \mathrm{eV}$ and Method 12 applied $10 \mathrm{eV}$ at ion funnel 1 (Table 1). LC-MS patterns displayed only slight changes, with Method 4 yielding 7141 peaks, 869 clusters, and 346 groups, and Method 12 producing 6614 peaks, 769 clusters, and 296 groups (Table 2 and 
Figure 3). Protein annotation by top-down sequencing was also comparable, as will be described in the next chapter.

\subsubsection{Charge State Preference Does Not Influence Precursor Selection}

Methods 9 and 11 (IS-CID + CID) only differed in their charge state preferred range. No charge state was specified in Method 11 (Table 1). In Method 9, a preference for the most abundant ions bearing 7-15 charges was applied to assess whether it would favour the selection of highly protonated ions for CID fragmentation. This parameter had no impact on the selection process of parent ions since both methods generated similar numbers of peaks, clusters and groups and, most importantly, an identical number of MS2 spectra (218) (Table 2). This indicated that the automatic MS2 mode of the Q-ToF aptly selected highly charged protein ions for subsequent CID fragmentation.

\subsubsection{A Long Duty Cycle Minimises MS/MS Events}

To maximise signal sensitivity and minimise background noise, high summation values were utilised, which resulted in duty cycles that lasted at least $2.7 \mathrm{~s}$ (MS1 only-Method 1) (Table 2). Furthermore, these duty cycles were extended upon MS/MS analyses in a method-specific fashion (from $5.5 \mathrm{~s}$ in Methods 4 and 12, $13.7 \mathrm{~s}$ in Methods 2-3 and 5-6, to $24.6 \mathrm{~s}$ in Methods 9-11), which restricted the number of full MS1 scans (Table 2). Consequently, it limited how many ions could be selected as parents for the CID process during the LC run.

Under our LC-MS/MS conditions, protein separation occurred from 2.5 to $25 \mathrm{~min}$ (22.5 min or $1350 \mathrm{~s}$ ) with two to four precursors selected per duty cycle. The maximum number of MS2 events were computed and reported in Table 2. Apart from the CID-only methods, which only realised 28\% (56/197 in Method 2) and 86\% (170/198 in Method 3) of their potential due to high threshold requirements, all the other methods delivered $99-100 \%$ of the expected number of MS2 events (Table 2). However, these numbers are low. Instruments of high resolution offering a faster scanning capability would therefore be extremely advantageous.

In our study, the slow scanning rate was partially alleviated by resorting to IS-CID only (Methods 4 and 12), whose fast fragmentation mode allowed for shorter duty cycles (5.5 s) and up to 247 scans during the 22.5-min separation (Table 2).

\subsubsection{High Energy Fragmentation Produces Lower $m / z$ Ions}

Overall, low-energy fragmentation was applied at the ion funnel 1 exit during the IS-CID MS mode $(3,5$ or $10 \mathrm{eV})$, except for three methods for which high energy was applied. Methods 5-7 applied 30,50 , and $70 \mathrm{eV}$, respectively (Table 1). The consequences of high-energy IS-CID become visible upon reaching $70 \mathrm{eV}$ (Method 7), which generates many more ions of less than $700 \mathrm{~m} / z$, indicative of protein fragmentation. For instance, in the mixed standard sample, Method 1 (MS1 only) resulted in 8728 peaks, with the vast majority above $700 \mathrm{~m} / z(8711,99.8 \%)$. Method $5(30 \mathrm{eV})$ produced 4509 peaks in total with only $6(0.1 \%)$ below $700 \mathrm{~m} / z$. Conversely, Method $7(70 \mathrm{eV})$ produced 3692 peaks in total, of which 93 (2.5\%) were of less than $700 \mathrm{~m} / z$ (Table 2).

Similarly, low-energy fragmentation $(10$ or $15 \mathrm{eV})$ was applied at the ion funnel 2 entrance during the IS-CID MS/MS mode, except for two methods for which greater energy was delivered. Methods 8 and 10 applied 20 and $30 \mathrm{eV}$, respectively (Table 1). Increasing the energy level during the IS-CID MS/MS step proved even more effective at fragmenting intact proteins within the ESI source, as more spectral peaks of low $m / z$ were created (Figure 3 ). For instance, in the mixed standard sample, applying $20 \mathrm{eV}$ (Method 8) or $30 \mathrm{eV}$ (Method 10) resulted in the proportion of peaks below $700 \mathrm{~m} / \mathrm{z}$ reaching $8.5 \%$ (281/3321 peaks) and 10.4\% (188/1816 peaks) for Methods 8 and 10, respectively (Table 2). This resulted in ions of lower $m / z$ being selected as precursors for a subsequent CID process. In the mixed standard sample, only three (1.5\%) MS2 spectra resulted from precursors of less than $700 \mathrm{~m} / \mathrm{z}$ using Method 5, while 20 (9.2\%) MS2 spectra were acquired from precursors of less than $700 \mathrm{~m} / \mathrm{z}$ 
using Method 10 (Table 2). However, as will be reported in the next chapter, such enhanced in-source fragmentation did not benefit the top-down sequencing output.

\subsubsection{CID Versus IS-CID}

While CID parameters mostly revolve around ion features (e.g., intensity threshold, charge state, number of precursors) that allow for relatively controlled and robust conditions, IS-CID parameters themselves pertain to factors operating globally within the ESI source and the ion transfer region, the most important one being the DC voltage applied, which affects signal sensitivity. Owing to its less controllable nature, the IS-CID fragmentation process is more complex and far less predictable than MS/MS results. Therefore, a prerequisite of IS-CID efficacy is good chromatographic resolution with little or no background contamination [16].

Our ESI-Q-ToF mass spectrometer establishes IS-CID fragmentation not within the ESI source per se but more accurately within the ion transfer area called funnel 2. The two funnel-staged ion transfer region separates the ions from the drying gas and solvent, as well as transfers these ions, with minimal losses, to the quadrupole stage. The first and second funnel stages are separated by a DC plate. By increasing the DC potentials of funnel 1, the ions are accelerated into funnel 2, which in turn activates IS-CID. This ingenious, albeit unpredictable, fragmentation mode was invented 30 years ago and reported to favour highly charged species at lower energetic collision (i.e., cone voltage) akin to conventional CID process [5]. As with CID, as the ions' $m / z$ increase, the IS-CID efficacy decreases. That was confirmed in our experiment. Parent ions were predominantly sampled from the $800-1300 \mathrm{~m} / \mathrm{z}$ range, where the most intense ions resolved, and only a handful of precursors arose from $1600 \mathrm{~m} / \mathrm{z}$ and above (Table 2).

IS-CID in combination with conventional CID on a ESI-Q-ToF system was tested at increasing cone voltage conditions (from 45 to $90 \mathrm{~V}$ ) by Ginter and colleagues (2004) to top-down sequence a mixture of seven protein standards; they reported that some proteins necessitated higher voltage than others for in-source fragmentation to occur [28]. In a different study where only IS-CID was applied to generate MS2 spectra using a ESI-Q-ToF instrument, source cone voltage varied from 20 to $70 \mathrm{~V}$ and was evaluated on a sample mixture of 13 protein standards [29]. The authors also concluded that IS-CID efficient fragmentation greatly depended on cone voltage; in their experience, $40-60 \mathrm{~V}$ proved the most optimal range.

\subsection{Top-Down Sequencing of Milk Proteins}

In the present study, a total of 11 MS/MS methods (Methods 2-12) were compared and the ultimate validation criterium was the coverage depth resulting from top-down sequencing annotation. The nine most abundant proteins known from milk were chosen to systematically assess AA sequence coverage across the 11 methods using Genedata Expressionist program. These proteoforms were $\alpha_{S 1}-\mathrm{CN}$ B-8P, $\alpha_{\mathrm{S} 2}$-CN A-11P, $\beta$-CN A1, $\beta$-CN A2, к-CN A-1P, k-CN B-1P, $\alpha$-LA B, $\beta$-LG A, and $\beta$-LG B. Table 3 documents the number of AAs top-down sequenced for each protein from either individual or mixed standards, or milk samples from Jerseys or Holsteins.

Results were consistent from sample to sample, particularly for the proteins that responded very well to top-down sequencing such as $\beta-\mathrm{CN} \mathrm{A} 2$ with a coefficient of variation $(\mathrm{CV})$ as low as $0.4 \%$.

For ease of interpretation, the values reported in Table 2 were converted to percentages and displayed as histograms (Figure 4). 
Table 3. Number of AAs top-down sequenced across the 11 MS2 methods from the nine most abundant milk proteins with the individual standards, mixed standards, and milk samples.

\begin{tabular}{|c|c|c|c|c|c|c|c|c|c|c|c|c|c|c|c|c|c|c|}
\hline Protein & Variant & PTM & Code & $\begin{array}{l}\text { Mo. Mass } \\
\text { (Da) }\end{array}$ & $\begin{array}{l}\text { Length } \\
\text { (AAs) }\end{array}$ & RT (min) & $\begin{array}{c}\text { Sample } \\
1\end{array}$ & $\begin{array}{l}\text { Method } \\
2\end{array}$ & $\begin{array}{l}\text { Method } \\
3\end{array}$ & $\begin{array}{l}\text { Method } \\
4\end{array}$ & $\begin{array}{l}\text { Method } \\
5\end{array}$ & $\begin{array}{l}\text { Method } \\
6\end{array}$ & $\begin{array}{c}\text { Method } \\
7\end{array}$ & $\begin{array}{c}\text { Method } \\
8\end{array}$ & $\begin{array}{l}\text { Method } \\
9\end{array}$ & $\begin{array}{c}\text { Method } \\
10\end{array}$ & $\begin{array}{c}\text { Method } \\
11\end{array}$ & $\begin{array}{l}\text { Method } \\
12\end{array}$ \\
\hline$\alpha_{\mathrm{S} 1}-\mathrm{CN}$ & B & $8 \mathrm{P}$ & $\alpha_{\mathrm{S} 1}-\mathrm{CN}$ B 8P & $23,600.3$ & 199 & $15-18$ & I & 196 & 196 & 195 & 194 & 195 & 190 & 70 & 195 & 7 & 194 & 195 \\
\hline$\alpha_{\mathrm{S} 2}-\mathrm{CN}$ & A & $11 \mathrm{P}$ & $\alpha_{\mathrm{S} 2}-\mathrm{CN}$ A 11P & $25,213.0$ & 207 & $6.8-8.3$ & I & 14 & 6 & 83 & 6 & 6 & 6 & 6 & 6 & 0 & 6 & 83 \\
\hline$\beta-\mathrm{CN}$ & A1 & $5 \mathrm{P}$ & $\beta-\mathrm{CN}$ A1 5P & $24,008.2$ & 209 & $21-22$ & I & 204 & 204 & 197 & 205 & 205 & 203 & 197 & 205 & 95 & 205 & 195 \\
\hline$\beta-\mathrm{CN}$ & A2 & $5 \mathrm{P}$ & $\beta-C N$ A2 5P & $23,968.2$ & 209 & $21.5-23$ & I & 202 & 202 & 201 & 203 & 203 & 201 & 186 & 203 & 92 & 203 & 200 \\
\hline k-CN & A & $1 \mathrm{P}$ & k-CN A 1P & $19,026.5$ & 169 & $6.5-8.5$ & I & 160 & 160 & 165 & 157 & 157 & 156 & 119 & 157 & 31 & 157 & 165 \\
\hline k-CN & B & $1 \mathrm{P}$ & K-CN B 1P & $18,993.6$ & 169 & $8.5-10$ & I & 162 & 162 & 157 & 160 & 159 & 157 & 133 & 161 & 59 & 161 & 154 \\
\hline$\alpha$-LA & B & & $\alpha$-LA B & $14,176.8$ & 123 & $15-18$ & I & 98 & 97 & 84 & 94 & 100 & 98 & 90 & 97 & 86 & 97 & 83 \\
\hline$\beta$-LG & A & & $\beta$-LG A & $18,355.5$ & 162 & $22-24$ & I & 162 & 162 & 161 & 155 & 155 & 153 & 153 & 162 & 151 & 162 & 161 \\
\hline$\beta$-LG & B & & $\beta$-LG B & $18,269.4$ & 162 & $20-22$ & I & 159 & 158 & 159 & 153 & 154 & 149 & 155 & 159 & 146 & 159 & 158 \\
\hline$\alpha_{S 1}-\mathrm{CN}$ & B & $8 \mathrm{P}$ & $\alpha_{\mathrm{S} 1}-\mathrm{CN}$ B $8 \mathrm{P}$ & $23,600.3$ & 199 & $15-18$ & $\mathrm{M}$ & 178 & 178 & 192 & 179 & 177 & 173 & 91 & 178 & 52 & 181 & 192 \\
\hline$\alpha_{\mathrm{S}_{2}}-\mathrm{CN}$ & A & $11 \mathrm{P}$ & $\alpha_{\mathrm{S}_{2}-\mathrm{CN}} \mathrm{A}$ 11P & $25,213.0$ & 207 & $6.8-8.3$ & M & 121 & 123 & 150 & 111 & 116 & 108 & 52 & 115 & 8 & 115 & 151 \\
\hline$\beta-\mathrm{CN}$ & A1 & $5 \mathrm{P}$ & $\beta-C N ~ A 15 P$ & $24,008.2$ & 209 & $21-22$ & $\mathrm{M}$ & 171 & 172 & 168 & 162 & 167 & 156 & 79 & 163 & 27 & 165 & 174 \\
\hline$\beta-\mathrm{CN}$ & A2 & $5 \mathrm{P}$ & $\beta-C N$ A2 5P & $23,968.2$ & 209 & $21.5-23$ & $\mathrm{M}$ & 201 & 201 & 201 & 203 & 201 & 200 & 182 & 202 & 43 & 203 & 201 \\
\hline k-CN & A & $1 \mathrm{P}$ & k-CN A 1P & $19,026.5$ & 169 & $6.5-8.5$ & $\mathrm{M}$ & 108 & 109 & 122 & 106 & 109 & 93 & 41 & 106 & 14 & 107 & 124 \\
\hline k-CN & B & $1 \mathrm{P}$ & K-CN B 1P & $18,993.6$ & 169 & $8.5-10$ & $\mathrm{M}$ & 122 & 125 & 112 & 120 & 120 & 110 & 33 & 122 & 10 & 121 & 116 \\
\hline$\alpha$-LA & B & & $\alpha$-LA B & $14,176.8$ & 123 & $15-18$ & $\mathrm{M}$ & 86 & 85 & 96 & 83 & 82 & 77 & 37 & 83 & 21 & 86 & 94 \\
\hline$\beta-L G$ & A & & $\beta$-LG A & $18,355.5$ & 162 & $22-24$ & M & 158 & 157 & 156 & 158 & 158 & 157 & 135 & 157 & 62 & 157 & 155 \\
\hline$\beta$-LG & B & & $\beta$-LG B & $18,269.4$ & 162 & $20-22$ & $\mathrm{M}$ & 111 & 112 & 126 & 106 & 107 & 96 & 42 & 106 & 20 & 111 & 125 \\
\hline$\alpha_{\mathrm{S} 1}-\mathrm{CN}$ & B & $8 \mathrm{P}$ & $\alpha_{\mathrm{S} 1}-\mathrm{CN}$ B $8 \mathrm{P}$ & $23,600.3$ & 199 & $15-18$ & $\mathrm{~J}$ & 194 & 195 & 198 & 195 & 195 & 189 & 137 & 194 & 97 & 195 & 198 \\
\hline$\alpha_{S 2}-\mathrm{CN}$ & A & $11 \mathrm{P}$ & $\alpha_{S_{2}-C N}$ A 11P & $25,213.0$ & 207 & $6.8-8.3$ & $\mathrm{~J}$ & 92 & 140 & 177 & 122 & 110 & 97 & 18 & 114 & 20 & 126 & 177 \\
\hline$\beta-\mathrm{CN}$ & A1 & $5 \mathrm{P}$ & $\beta-C N$ A1 5P & $24,008.2$ & 209 & $21-22$ & $\mathrm{~J}$ & 206 & 206 & 205 & 206 & 206 & 205 & 191 & 206 & 110 & 206 & 204 \\
\hline$\beta-C N$ & A2 & $5 \mathrm{P}$ & $\beta-C N$ A2 5P & $23,968.2$ & 209 & $21.5-23$ & $\mathrm{~J}$ & 199 & 199 & 201 & 200 & 200 & 199 & 188 & 199 & 106 & 199 & 201 \\
\hline k-CN & $\mathrm{A}$ & $1 \mathrm{P}$ & K-CN A $1 P$ & $19,026.5$ & 169 & $6.5-8.5$ & J & 82 & 132 & 151 & 121 & 107 & 104 & 12 & 110 & 30 & 119 & 154 \\
\hline k-CN & B & $1 \mathrm{P}$ & K-CN B 1P & $18,993.6$ & 169 & $8.5-10$ & $\mathrm{~J}$ & 164 & 164 & 157 & 164 & 163 & 164 & 146 & 164 & 78 & 163 & 157 \\
\hline$\alpha$-LA & B & & $\alpha$-LA B & $14,176.8$ & 123 & $15-18$ & $\mathrm{~J}$ & 120 & 120 & 121 & 120 & 120 & 111 & 62 & 120 & 38 & 120 & 121 \\
\hline$\beta$-LG & A & & $\beta$-LG A & $18,355.5$ & 162 & $22-24$ & $\mathrm{~J}$ & 154 & 154 & 153 & 154 & 154 & 154 & 139 & 154 & 73 & 154 & 153 \\
\hline$\beta$-LG & B & & $\beta$-LG B & $18,269.4$ & 162 & $20-22$ & $\mathrm{~J}$ & 158 & 156 & 156 & 158 & 156 & 154 & 134 & 156 & 76 & 156 & 155 \\
\hline$\alpha_{S 1-C N}$ & B & $8 \mathrm{P}$ & $\alpha_{\mathrm{S} 1}-\mathrm{CN}$ B 8P & $23,600.3$ & 199 & $15-18$ & $\mathrm{H}$ & 191 & 191 & 197 & 183 & 184 & 177 & 133 & 185 & 89 & 183 & 197 \\
\hline$\alpha_{S 2}-\mathrm{CN}$ & A & $11 \mathrm{P}$ & 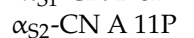 & $25,213.0$ & 207 & $6.8-8.3$ & $\mathrm{H}$ & 172 & 181 & 192 & 179 & 179 & 172 & 65 & 178 & 88 & 180 & 193 \\
\hline$\beta-\mathrm{CN}$ & $\mathrm{A} 1$ & $5 \mathrm{P}$ & $\beta-\mathrm{CN}$ A1 5P & $24,008.2$ & 209 & $21-22$ & $\mathrm{H}$ & 204 & 204 & 200 & 204 & 204 & 204 & 181 & 204 & 170 & 204 & 201 \\
\hline$\beta-\mathrm{CN}$ & A2 & $5 \mathrm{P}$ & $\beta-C N$ A2 5P & $23,968.2$ & 209 & $21.5-23$ & $\mathrm{H}$ & 206 & 207 & 203 & 207 & 207 & 207 & 198 & 207 & 168 & 207 & 203 \\
\hline $\mathrm{k}-\mathrm{CN}$ & A & $1 \mathrm{P}$ & $\mathrm{k}-\mathrm{CN}$ A $1 \mathrm{P}$ & $19,026.5$ & 169 & $6.5-8.5$ & $\mathrm{H}$ & 147 & 150 & 165 & 147 & 153 & 133 & 54 & 151 & 72 & 150 & 165 \\
\hline $\mathrm{k}-\mathrm{CN}$ & B & $1 \mathrm{P}$ & k-CN B 1P & $18,993.6$ & 169 & $8.5-10$ & $\mathrm{H}$ & 89 & 97 & 118 & 91 & 86 & 82 & 11 & 89 & 17 & 95 & 118 \\
\hline$\alpha$-LA & B & & $\alpha$-LA B & $14,176.8$ & 123 & $15-18$ & $\mathrm{H}$ & 118 & 119 & 119 & 106 & 105 & 94 & 52 & 107 & 23 & 104 & 119 \\
\hline$\beta$-LG & $\mathrm{A}$ & & $\beta$-LG A & $18,355.5$ & 162 & $22-24$ & $\mathrm{H}$ & 157 & 159 & 156 & 158 & 158 & 155 & 136 & 156 & 97 & 157 & 155 \\
\hline$\beta$-LG & B & & $\beta$-LG B & $18,269.4$ & 162 & $20-22$ & $\mathrm{H}$ & 162 & 162 & 159 & 162 & 162 & 160 & 138 & 162 & 128 & 162 & 158 \\
\hline
\end{tabular}

I, individual standard; M, mixed standard; J, Jersey milk; H, Holstein milk. 


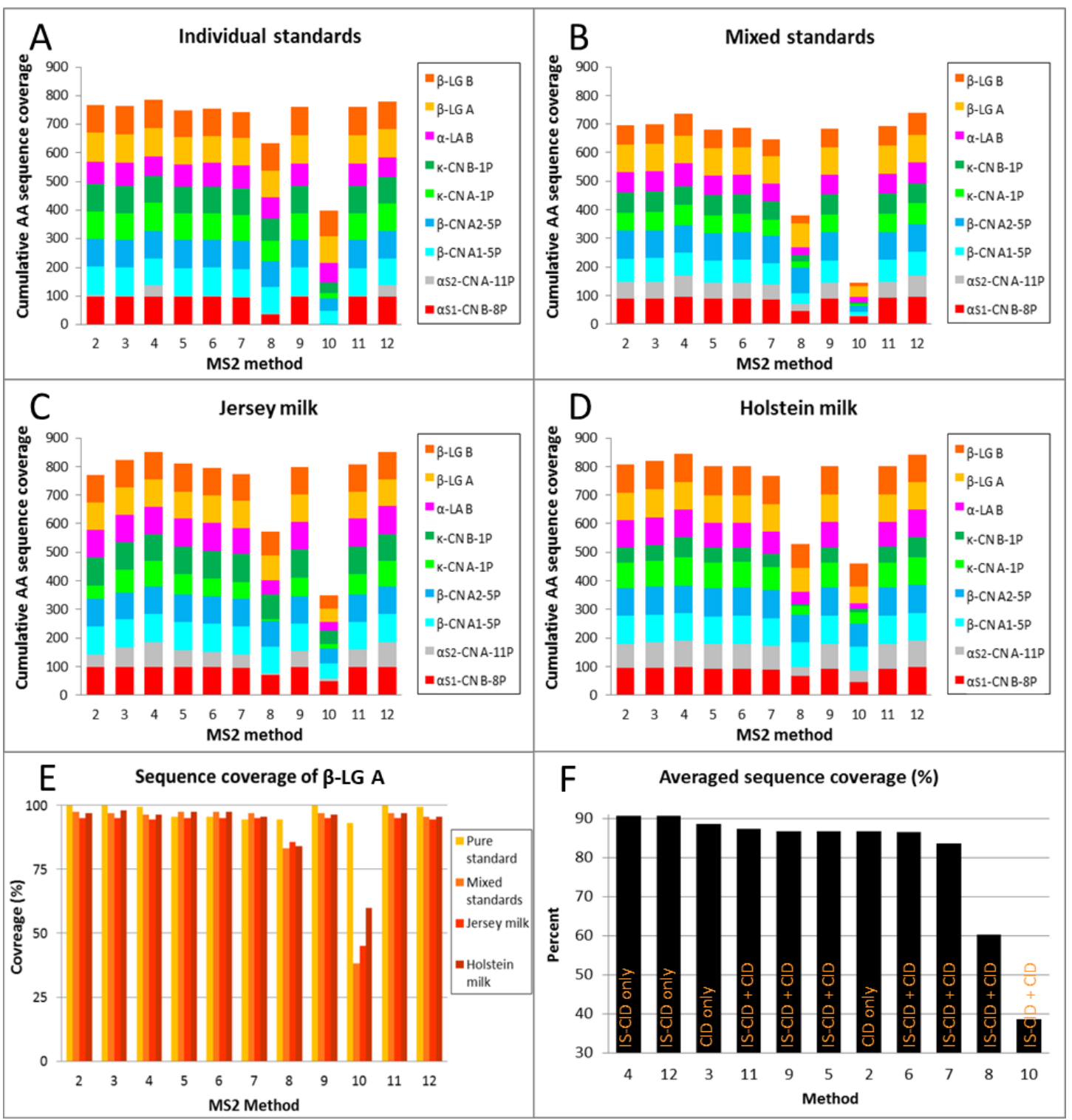

Figure 4. Comparison of the $11 \mathrm{MS} / \mathrm{MS}$ methods based on the AA sequence coverage in percent of the nine most prominent milk proteins. (A) Cumulative coverage in the individual standards. (B) Cumulative coverage in the mixed standards. (C) Cumulative coverage in the Jersey milk sample. (D) Cumulative coverage in the Holstein milk sample. (E) Illustration of AA sequence coverage for one particular protein, $\beta$-LG A, in increasingly complex samples. (F) Averaged sequence coverage for all eight proteins sorted by method efficacy; the fragmentation modes are reported as well.

\subsubsection{Reproducibility and Protein Specificity}

While being both protein- and method-specific, top-down sequencing results were reproducible across samples of increasing complexity, from the least (individual standards, Figure 4A) and mildly (mixed standards, Figure 4B) complex, to the most complex samples (raw milk, Figure 4C-D). The sample reproducibility was also demonstrated on one particular protein, $\beta$-LG A, which exhibited CV values spanning from 1.0\% (Method 7) to 1.9\% (Methods 9 and 12), omitting Methods 8 and 10 , which were not only the least efficient but also the least reproducible (Figure 4E). As observed in previous studies [28,29], top-down sequencing success is protein-dependent. In our conditions, $\alpha_{\mathrm{S} 2}-\mathrm{CN}$ A-10P was the least responsive, possibly due to its elevated number of phosphorylation sites, while $\beta$-LGs and $\beta$-CNs were the most responsive. 


\subsubsection{Low-Energy Fragmentation Drives Top-Down Sequencing Efficacy}

The histograms in Figure 4 also clearly demonstrate which methods were suitable for top-down sequencing and which were not. In Figure $4 \mathrm{~F}$, the methods have been sorted in descending order of AA sequence coverage. Under our conditions, the best methods were Methods 4 and 12 (90.6\% sequence coverage on average), closely followed by Methods $3(88.6 \%), 11(87.4 \%), 9(86.8 \%), 5$ and 2 $(86.7 \%), 6(86.6 \%)$, and $7(83.6 \%)$. The worst-performing methods were $8(60.2 \%)$ and $10(38.6 \%)$. Based on our observations, we can conclude that the methods that resorted to IS-CID on its own with little energy applied, in combination with CID or not, were best suited to top-down sequence prominent milk proteins.

Optimal conditions in our case involved applying $3 \mathrm{eV}$ (Method 4) or $10 \mathrm{eV}$ (Method 12) to the ion funnel 1 exit during the IS-CID MS step and $15 \mathrm{eV}$ to the ion funnel 2 entrance during the IS-CID MS/MS step. What seemed to be detrimental for our purposes was using too much energy $(20 \mathrm{eV}$ in Method 8 and $30 \mathrm{eV}$ in Method 10) during the IS-CID MS/MS step. Moreover, we do not recommend applying an energy level higher than $10 \mathrm{eV}$ during the IS-CID MS step, as was tested in Methods 5 $(30 \mathrm{eV}), 6(50 \mathrm{eV})$, and $7(70 \mathrm{eV})$.

\subsubsection{AA Position Has No Influence on Fragmentation}

In an attempt to further explain how CID-based fragmentation operated under our conditions, we aligned side by side all the AA sequences of the nine proteins of interest for each of the $11 \mathrm{MS} / \mathrm{MS}$ methods and blackened the AAs that were successfully top-down sequenced. For each protein, the sequenced AAs were counted across the methods and, as a result, we generated a score from 0 (not sequenced at all) to 11 (sequenced in all methods). Based on this scoring system, the corresponding position in the sequence was coloured red (the higher the score, the darker the shade). This is represented in Supplementary Figure S1. This display allowed for the quick visualisation of regions that remained unfragmented under our various conditions. Such recalcitrant regions were scattered along the protein sequence, suggesting that the AA position had no influence over fragmentation efficiency.

All cow's milk caseins are heavily phosphorylated. There was no visible pattern associated with phosphorylation sites (highlighted in yellow) either, apart from $\alpha_{S 2}-\mathrm{CN}$ A-10P, for which five phosphorylated serine residues ( $\mathrm{S}$ at positions $7,13,31,56$, and 143) resisted fragmentation. PTMs were not investigated in this study. Milk proteins are also glycosylated, as has been well documented for k-CN, for instance [36]. Glycosylation sites could account for areas difficult to fragment, but we could not test this hypothesis in the present study.

\subsubsection{Hydrophobicity Affects Fragmentation}

To further elucidate whether AAs themselves influenced fragmentation efficiency, we converted the AA counts from Supplementary Figure S1 into percentages, sorted them according to their highest fragmentation efficiency (meaning sequenced across all 11 MS/MS methods) and plotted them (Supplementary Figure S2). Underneath the chart we listed the physical properties attributed to AAs (ThermoFisher Scientific website) to assist us in finding a pattern.

In this representation, leucine, glycine, cysteine, tryptophan, histidine, and isoleucine displayed the best response to top-down sequencing with a success rate above 17\% across $11 \mathrm{MS} / \mathrm{MS}$ methods (Supplementary Figure S2). These AAs are moderately to highly hydrophobic. Conversely, threonine, asparagine and arginine showed the lowest success rate (less than $9 \%$ across the 11 methods). Such AAs are hydrophilic. Our results suggest that AA hydrophobicity level has an impact on fragmentation efficiency.

\subsubsection{High Sequence Coverage Is Critical for Allelic Variants}

High sequence coverage is a prerequisite to successfully distinguishing between protein allelic variants such as $\beta-\mathrm{CN} A 1$ and $\mathrm{A} 2$, which only vary at position $67(\mathrm{H}$ to $\mathrm{P}) ; \mathrm{k}-\mathrm{CN} \mathrm{A}$ and $\mathrm{B}$, which 
vary at positions 136 ( $\mathrm{T}$ to I) and 148 (D to A); and $\beta$-LG A and B, which vary at positions 64 (D to G) and 118 (V to A). The mixed standard sample, Method 10, and, in the case of $\mathrm{k}$-CNs, Method 8 failed to deliver such relevant information since AAs at the aforementioned positions were not sequenced (Supplementary Figure S1).

Interestingly, some genetic variants proved more amenable to top-down sequencing than others. For instance, in the mixed standard sample processed with Method 12, $\beta-\mathrm{CN} \mathrm{A2} \mathrm{(97 \% )} \mathrm{was} \mathrm{more}$ thoroughly sequenced than $\beta-\mathrm{CN}$ A1 (83\%). Likewise, $\beta$-LG A (96\%) displayed greater sequence coverage than $\beta$-LG B (77\%); this was also observed for $\mathrm{k}-\mathrm{CN}$ A $(73 \%)$ and $\mathrm{k}-\mathrm{CN}$ B (68\%) (Table 3 and Figure S1). In Holstein milk samples, we managed to reach $100 \%$ sequence coverage of $\beta$-LG B in six out of $11 \mathrm{MS} / \mathrm{MS}$ methods. Complete top-down sequencing was also achieved for $\beta$-LG A in individual standard samples in four MS/MS methods (Table 3).

In the early days of top-down proteomics on ESI-Q-ToF platforms, the resulting short sequence tags from $\mathrm{C}$ - and $\mathrm{N}$-termini from small to medium-sized proteins were just long enough to unambiguously identify the protein family, but not their allelic variants [26-30]. Almost complete top-down sequencing was achieved in 2009 with $17 \mathrm{kDa}$ horse myoglobin and $30 \mathrm{kDa}$ bovine carbonic anhydrase II being $92 \%$ and $96 \%$ sequenced, respectively [31]. Since then and to our knowledge, Q-ToF instruments have not been employed for top-down sequencing of intact proteins from complex biological matrices because they have been superseded by more expensive platforms such as FT mass spectrometers, offering better power resolution and alternative fragmentation methods like ETD, HCD, and ECD.

\section{Materials and Methods}

\subsection{Materials and Sample Preparation}

The experimental design is schematised in Figure 1. Protein standards purchased from Sigma-Aldrich (Castle Hill, NSW, Australia) were alpha-casein ( $\alpha-\mathrm{CN})$ from bovine milk (C6780-250MG, $70 \%$ pure), beta-casein ( $\beta-\mathrm{CN}$ ) from bovine milk (C6905-250MG, 98\% pure), kappa-casein ( $\mathrm{k}-\mathrm{CN})$ from bovine milk (C0406-250MG, 70\% pure), alpha-lactalbumin ( $\alpha$-LA) from bovine milk (L5385-25MG, 85\% pure), and beta-lactoglobulin ( $\beta$-LG) from bovine milk (L3908-250MG, 90\% pure). These lyophilised standards were prepared as described in [23]. Briefly, they were fully solubilised at a $10 \mathrm{mg} / \mathrm{mL}$ concentration in 50\% MilliQ (MerckMillipore, Bayswater, VIC, Australia) water/50\% solution A (0.1 M Bis-Tris, $6 \mathrm{M}$ Guanidine- $\mathrm{HCl}, 5.37 \mathrm{mM}$ sodium citrate tribasic dehydrate, and $20 \mathrm{mM}$ DTT). A volume of $50 \%$ acetic acid to reach $1 \%$ acetic acid final concentration was added to the standards. A $0.1-\mathrm{mL}$ aliquot of the solubilised standard was transferred into a $100-\mu \mathrm{L}$ glass insert placed in a 2-mL glass vial for immediate analysis by LC-MS. A standard mixture was prepared by mixing individual standards in the following proportions to account for various ionisation efficiency [23]: $25 \% \alpha-\mathrm{CN}, 25 \% \mathrm{~K}-\mathrm{CN}, 20 \%$ $\beta$-LG, 20\% $\beta$-CN, and 10\% $\alpha$-LA.

Milk collection from Holstein-Friesian cows (coded H) and Jersey cows (coded J) was described in [35]. Milk sample preparation was described in [23]. Briefly, $0.5 \mathrm{~mL}$ of cold skim milk was transferred into a $1.5-\mathrm{mL}$ tube and $0.5 \mathrm{~mL}$ of Solution A was added. A $0.02 \mathrm{~mL}$ volume of $50 \%$ acetic acid $(1 \%$ acetic acid final concentration, $\mathrm{pH}$ 5.8) was then added. A 0.1-mL aliquot of the milk protein extract was transferred into a $100-\mu \mathrm{L}$ glass insert placed in a $2-\mathrm{mL}$ glass vial for immediate analysis by LC-MS.

\subsection{HPLC Separation of Intact Proteins}

The separation of intact proteins by UHPLC 1290 Infinity Binary LC system (Agilent) through a Aeris $^{\mathrm{TM}}$ WIDEPORE XB-C8 (3.6 $\mu \mathrm{m}$ particle size, $200 \AA$ pore size, $150 \times 2.1 \mathrm{~mm}$ dimensions, C8 reverse phase core-shell silica from Phenomenex (Lane Cove, NSW, Australia) column at $75{ }^{\circ} \mathrm{C}$ was described in [23]. In brief, $3 \mu \mathrm{L}$ of sample was injected and separated as followed: starting conditions $20 \% \mathrm{~B}$, ramping to $28 \% \mathrm{~B}$ in $2.5 \mathrm{~min}$, ramping to $40 \% \mathrm{~B}$ in $27.5 \mathrm{~min}$, ramping to $99 \% \mathrm{~B}$ in $1 \mathrm{~min}$ and held for $4 \mathrm{~min}$, lowering to $20 \% \mathrm{~B}$ in $0.1 \mathrm{~min}$, equilibration at $20 \%$ B for $4.9 \mathrm{~min}$. Mobile phase flow 
rates was $200 \mu \mathrm{L} / \mathrm{min}$. Mobile phase A contained ACN with $0.1 \% \mathrm{FA}$ and $0.02 \%$ trifluoroacetic acid (TFA), and mobile phase B contained $\mathrm{H} 2 \mathrm{O}$ with $0.1 \%$ FA and $0.02 \%$ TFA. The diode array detector (DAD) spectrum was acquired from 190 to $400 \mathrm{~nm}$. The pressure limit was set at 600 bars.

\subsection{MS1 Analysis}

MS1 analysis was described in [23]. The UHPLC was on-line with a maXis HD UHR-Q-ToF (60,000 resolution) fitted with a standard ESI Apollo-source (BrukerDaltonikGmbh, Preston, VIC, Australia). To ensure mass accuracy, a Na-formate solution was infused continuously at $0.1 \mathrm{~mL} / \mathrm{h}$ and the first $2.5 \mathrm{~min}$ of each run were used to re-calibrate masses post-acquisition. Each 40 -min run was thus segmented as follows: $2.5 \mathrm{~min}$ to waste and the following $37.5 \mathrm{~min}$ to source. Capillary voltage was set at $4500 \mathrm{~V}$. The nebuliser was set at $1.5 \mathrm{bar}$. The dry gas was set at $8 \mathrm{~L} / \mathrm{min}$. The dry temperature was set at $190^{\circ} \mathrm{C}$. The transfer funnel RF and multipole RF were set at $400 \mathrm{Vpp}$; no IS-CID energy was applied. The quadrupole ion energy was $5 \mathrm{eV}$, the collision cell energy was $10 \mathrm{eV}$, and the collision RF $1800 \mathrm{Vpp}$. The ion cooler transfer time was $120 \mu \mathrm{s}$, with a prepulse storage of $10 \mu \mathrm{s}$ and a RF of $400 \mathrm{Vpp}$. The ion polarity was positive and scan mode was MS. The rolling average mode was activated and set at 2. This MS1 method is called Method 1 hereafter.

\subsection{MS2 Analyses and Top-Down Sequencing}

MS/MS experiments were performed using the same LC parameters and MS1 parameters on the Q-TOF mass spectrometer as described above. A total of 11 MS2 methods were tested; their parameters are indicated in Table 1. These methods are referred to as Method 2 to Method 12 hereafter. LC-MS files were visualised using Bruker Compass DataAnalysis version 4.2. MS2 spectra were annotated using Bruker Biotools version 3.2 and SequenceEditor version 3.2. The retrieval of AA sequences was detailed in [23].

\subsection{Protein Annotation in Genedata Expressionist}

The data files obtained following LC-MS analysis using MS2 methods 2 to 12 were curated in the Refiner MS module of Genedata Expressionist ${ }^{\circledR}$ version 11.0 with the following parameters: (1) Conversion of MS/MS to primary MS. (2) Chromatogram chemical noise subtraction using moving average algorithm and a five-scan window. (3) Spectrum smoothing using a moving average algorithm and a five-point $m / z$ window. (4) Chromatogram peak detection using a five-scan summation window, a minimum peak size of four scans with boundaries merge strategy and five-point maximum merge distance, a curvature-based peak detection with $70 \%$ intensity threshold and inflection points boundary determination. (5) Chromatogram isotope clustering using a $0.2 \mathrm{~min}$ and $20 \mathrm{ppm}$ tolerances, a peptide isotope shaping with 1-8 protons, a 0.6 log-ration maximum distance, mono-isotopic computation, linear charge dependency with a minimum size ratio of 0.65. (6) Singleton filtering. (7) Peptide mapping with a 50 ppm tolerance, unspecific enzyme, three missed cleavage maximum, 10 AAs minimum length, and variable modifications, as explained hereafter. The peptide mapping was performed using a text file containing all 49 AA sequences in FASTA format of the bovine allelic variants of milk caseins, alpha-lactalbumin, and beta-lactoglobulin (Farrell et al., 2004) with the following variable modifications: pyro-Glu (N-term Q) and Phospho (ST). Some aspects of this workflow are illustrated in Figure S3.

Protein deconvolution was also performed on Method 1 files in the Refiner MS module of Genedata Expressionist ${ }^{\circledR}$ version 11.0 with the following parameters. (1) Data sweep to remove UV data. (2) Chromatogram chemical noise subtraction using moving average algorithm, with a subtraction method, a 71 scan window, and $60 \%$ quantile. (3) Chromatogram lock mass using the Na-formate ion series. (4) Retention time range restriction from 4 to $25 \mathrm{~min}$. (5) Intact protein activity using the harmonic suppression deconvolution method with 0.02 Da steps and 5-30 kDa masses. (6) Intensity thresholding using a clipping method with an intensity of 50. (7) Spectrum baseline 
subtraction using a $99 \%$ quantile and $30 \mathrm{kDa} \mathrm{m} / \mathrm{z}$ window. (8) Chromatogram retention time alignment using a pairwise alignment-based tree scheme with 50-scan search interval and a gap penalty of 1 .

All MS1 and MS2 files are available from the stable public repository MassIVE at the following URL: http:/ / massive.ucsd.edu/ProteoSAFe/datasets.jsp with the accession number MSV000082070.

\subsection{Validation and Computational Analyses}

Top-down sequencing results were visualised and validated in Genedata Expressionist ${ }^{\circledR}$ version 11.0 Refiner MS module within the Peptide Mapping activity and all its subsidiary tabs as well as using Bruker Biotools version 3.2 and SequenceEditor version 3.2, as exemplified in Figure S3.

Top-down sequencing annotations (including b- and y-ions series) for each of the nine proteins of interest in every sample and each of the 11 MS/MS methods were exported from Refiner MS module as .csv files. The .csv files contained the list of peptides identified for each protein along with the peptide AA lengths and their position in the protein sequence they matched to. The .csv files were imported into Microsoft ${ }^{\circledR}$ Excel 2016 software for further processing. Coverage information was derived from the length and position of the identified peptides relative to their matching protein sequence to produce Table 3 and Figure 4. AA responsiveness to MS2 fragmentation was obtained using the positions of the first and last AA of the identified peptides. These analyses produced Figures S1 and S2.

\section{Conclusions}

In this experiment, we have fine-tuned IS-CID parameters with and without traditional CID using a LC-ESI-Q-ToF system in order to optimise the top-down sequencing of milk intact proteins. The most efficient methods utilised IS-CID on its own at low energy. Full $(100 \%)$ sequence covering could be obtained on $\beta$-LGs, and overall enough coverage was achieved to distinguish between allelic proteoforms, varying only by one or two AAs. Such information is highly biologically relevant as alleles influence function and phenotypic features. In bovine milk, for instance, 12 allelic variants of beta-caseins have been identified across different breeds and populations [37]. The most common variants, A1 and A2, differ at amino acid position 67 with histidine in A1 and proline in A2 milk, as a result of a single nucleotide difference. One application of this research would be screening for the presence of the A1 variant in cow's milk samples.

Supplementary Materials: The following are available online, Figure S1: Top-down sequencing responsiveness of AAs for each of the eight most abundant proteins from the mixed standard sample, Figure S2: CID-friendly amino acids from top-down sequenced milk proteins, Figure S3: Data processing of MS/MS files using Genedata Refiner and DataAnalysis/Biotools exemplified on the Holstein milk sample processed using Method 3.

Author Contributions: Conceptualisation, D.V.; methodology, D.V. and D.M.; software, D.V. and D.M.; validation, D.V.; formal analysis, D.V.; investigation, D.V.; resources, S.R.; data curation, D.V.; writing-original draft preparation, D.V.; writing-review and editing, D.V., D.M., and S.R.; visualisation, D.V.; supervision, S.R.; project administration, D.V.; funding acquisition, S.R.

Funding: This research received no external funding.

Conflicts of Interest: The authors declare no conflict of interest.

\section{Abbreviations}

$\begin{array}{ll}\text { Abbreviation } & \text { Definition } \\ \text { AA } & \text { Amino acid } \\ \text { ACN } & \text { Acetonitrile } \\ \text { AE } & \text { Active exclusion } \\ \text { CAD } & \text { Collisionally activated dissociation } \\ \text { CID } & \text { Collision-induced dissociation } \\ \text { CV } & \text { Coefficient of variation }\end{array}$




$\begin{array}{ll}\text { DAD } & \text { Diode array detector } \\ \text { DC } & \text { Direct current } \\ \text { ECD } & \text { Electron capture dissociation } \\ \text { ESI } & \text { Electrospray ionisation } \\ \text { ETD } & \text { Electron transfer dissociation } \\ \text { eV } & \text { Electron-volt } \\ \text { FA } & \text { Formic acid } \\ \text { FT } & \text { Fourier transform } \\ \text { HCD } & \text { Higher-energy collisional dissociation } \\ \text { HPLC } & \text { High-performance liquid chromatography } \\ \text { IS-CID } & \text { In-source CID } \\ \text { kDa } & \text { KiloDalton } \\ \text { keV } & \text { Kiloelectron-volt } \\ \text { LC-MS } & \text { Liquid chromatography-mass spectrometry } \\ \text { LIT } & \text { Linear ion trap } \\ m / z & \text { Mass-to-charge ratio } \\ \text { MS } & \text { Mass spectrometry } \\ \text { MS/MS } & \text { Tandem MS } \\ \text { MS1 } & \text { Full scan MS } \\ \text { MS2 } & \text { Tandem MS } \\ \text { MW } & \text { Molecular weight } \\ \text { ppm } & \text { Parts per million } \\ \text { PTM } & \text { Post-translational modification } \\ \text { Q } & \text { Quadrupole } \\ \text { QQQ } & \text { Triple quadrupole } \\ \text { Q-ToF } & \text { Quadrupole time-of-flight } \\ \text { RF } & \text { Radiofrequency } \\ \text { TFA } & \text { Trifluoroacetic acid } \\ \text { ToF } & \text { Time-of-flight } \\ \text { UHT } & \text { Ultra-high temperature } \\ \text { V } & \text { Volt } \\ \alpha-L A & \text { Alpha-lactalbumin } \\ \alpha \text { S1-CN } & \text { Alpha-S1-casein } \\ \alpha \text { S2-CN } & \text { Alpha-S2-casein } \\ \beta-C N ~ & \text { Beta-casein } \\ \text { k-CG } & \text { Kappasein } \\ & \end{array}$

\section{References}

1. Kelleher, N.L.; Lin, H.Y.; Valaskovic, G.A.; Aaserud, D.J.; Fridriksson, E.K.; McLafferty, F.W. Top down versus bottom up protein characterization by tandem high-resolution mass spectrometry. J. Am. Chem. Soc. 1999, 121, 806-812. [CrossRef]

2. Toby, T.K.; Fornelli, L.; Kelleher, N.L. Progress in Top-Down Proteomics and the Analysis of Proteoforms. Annu. Rev. Anal. Chem. 2016, 9, 499-519. [CrossRef] [PubMed]

3. Dole, M.; Mack, L.L.; Hines, R.L.; Mobley, R.C.; Ferguson, L.D.; Alice, M.B. Molecular Beams of Macroions. J. Chem. Phys. 1968, 49, 2240-2249. [CrossRef]

4. Fenn, J.B.; Mann, M.; Meng, C.K.; Wong, S.F.; Whitehouse, C.M. Electrospray ionization for mass spectrometry of large biomolecules. Science 1989, 246, 64-71. [CrossRef] [PubMed]

5. Loo, J.A.; Udseth, H.R.; Smith, R.D. Collisional Effects on the Charge Distribution of Ions from Large Molecules, Formed by Electrospray-ionization Mass Spectrometry. Rapid Commun. Mass Spectrom. 1988, 2, 207-210. [CrossRef]

6. Mann, M.; Meng, C.K.; Fenn, J.B.F. Interpreting Mass Spectra of Multiply Charged Ions. Anal. Chem. 1989, 61, 1702-1708. [CrossRef] 
7. Jones, A.W.; Cooper, H.J. Dissociation techniques in mass spectrometry-based proteomics. Analyst 2011, 136, 3419-3429. [CrossRef] [PubMed]

8. McLafferty, F.W.; Bryce, T.A. Metastable-ion characteristics: Characterization of isomeric molecules. Chem. Commun. 1967, 0, 1215-1217. [CrossRef]

9. Jennings, K.R. Collision-induced decompositions of aromatic molecular ions. Int. J. Mass Spectrom. Ion. Phys. 1968, 1, 227-235. [CrossRef]

10. Garcia, B.A. What does the future hold for Top Down mass spectrometry? J. Am. Soc. Mass Spectrom. 2010, 21, 193-202. [CrossRef] [PubMed]

11. Pamreddy, A.; Panyala, N.R. Top-down proteomics: Applications, recent developments and perspectives. J. Appl.'Bioanal. 2016, 2, 52-75. [CrossRef]

12. Katta, V.; Chowdhury, S.K.; Chait, B.T. Use of a single-quadrupole mass spectrometer for collision-induced dissociation studies of multiply charged peptide ions produced by electrospray ionization. Anal. Chem. 1991, 63, 174-178. [CrossRef] [PubMed]

13. Bure, C.; Lange, C. Comparison of dissociation of ions in an electrospray source, or a collision cell in tandem mass spectrometry. Curr. Org. Chem. 2003, 7, 1613-1624. [CrossRef]

14. Van Dongen, W.D.; van Wijk, J.I.; Green, B.N.; Heerma, W.; Haverkamp, J. Comparison between collision induced dissociation of electrosprayed protonated peptides in the up-front source region and in a low-energy collision cell. Rapid Commun. Mass Spectrom. 1999, 13, 1712-1716. [CrossRef]

15. Senko, M.W.; Beu, S.C.; McLafferty, F.W. High-resolution tandem mass spectrometry of carbonic anhydrase. Anal. Chem. 1994, 66, 415-418. [CrossRef] [PubMed]

16. Parcher, J.F.; Wang, M.; Chittiboyina, A.G.; Khan, I.A. In-source collision-induced dissociation (IS-CID): Applications, issues and structure elucidation with single-stage mass analyzers. Drug Test. Anal. 2018, 10, 28-36. [CrossRef] [PubMed]

17. Kelleher, N.L. Top-down proteomics. Anal. Chem. 2004, 76, 197A-203A. [CrossRef] [PubMed]

18. Loo, J.A.; Edmonds, C.G.; Smith, R.D. Primary sequence information from intact proteins by electrospray ionization tandem mass spectrometry. Science 1990, 248, 201-204. [CrossRef] [PubMed]

19. Loo, J.A.; Quinn, J.P.; Ryu, S.I.; Henry, K.D.; Senko, M.W.; McLafferty, F.W. High-resolution tandem mass spectrometry of large biomolecules. Proc. Natl. Acad. Sci. USA 1992, 89, 286-289. [CrossRef] [PubMed]

20. Beu, S.C.; Senko, M.W.; Quinn, J.P.; McLafferty, F.W. Improved fourier-transform ion-cyclotron-resonance mass spectrometry of large biomolecules. J. Am. Soc. Mass Spectrom. 1993, 4, 190-192. [CrossRef]

21. Shevchenko, A.; Chernushevich, I.; Ens, W.; Standing, K.G.; Thomson, B.; Wilm, M.; Mann, M. Rapid 'de novo' peptide sequencing by a combination of nanoelectrospray, isotopic labeling and a quadrupole/time-of-flight mass spectrometer. Rapid Commun. Mass Spectrom. 1997, 11, 1015-1024. [CrossRef]

22. Morris, H.R.; Paxton, T.; Dell, A.; Langhorne, J.; Berg, M.; Bordoli, R.S.; Hoyes, J.; Bateman, R.H. High sensitivity collisionally-activated decomposition tandem mass spectrometry on a novel quadrupole/ orthogonal-acceleration time-of-flight mass spectrometer. Rapid Commun. Mass Spectrom. 1996, 10, 889-896. [CrossRef]

23. Vincent, D.; Elkins, A.; Condina, M.R.; Ezernieks, V.; Rochfort, S. Quantitation and Identification of Intact Major Milk Proteins for High-Throughput LC-ESI-Q-TOF MS Analyses. PLoS ONE 2016, 11, e0163471. [CrossRef] [PubMed]

24. Domon, B.; Aebersold, R. Mass spectrometry and protein analysis. Science 2006, 312, 212-217. [CrossRef] [PubMed]

25. Steen, H.; Kuster, B.; Mann, M. Quadrupole time-of-flight versus triple-quadrupole mass spectrometry for the determination of phosphopeptides by precursor ion scanning. J. Mass Spectrom. 2001, 36, 782-790. [CrossRef] [PubMed]

26. Nemeth-Cawley, J.F.; Rouse, J.C. Identification and sequencing analysis of intact proteins via collision-induced dissociation and quadrupole time-of-flight mass spectrometry. J. Mass Spectrom. 2002, 37, 270-282. [CrossRef] [PubMed]

27. Nemeth-Cawley, J.F.; Tangarone, B.S.; Rouse, J.C. “Top Down” characterization is a complementary technique to peptide sequencing for identifying protein species in complex mixtures. J. Proteome Res. 2003, 2, 495-505. [CrossRef] [PubMed] 
28. Ginter, J.M.; Zhou, F.; Johnston, M.V. Generating protein sequence tags by combining cone and conventional collision induced dissociation in a quadrupole time-of-flight mass spectrometer. J. Am. Soc. Mass Spectrom. 2004, 15, 1478-1486. [CrossRef] [PubMed]

29. Johnson, R.W.J.; Ahmed, T.F.; Miesbauer, L.J.; Edalji, R.; Smith, R.; Harlan, J.; Dorwin, S.; Walter, K.; Holzman, T. Protein fragmentation via liquid chromatography-quadrupole time-of-flight mass spectrometry: The use of limited sequence information in structural characterization. Anal. Biochem. 2005, 341, 22-32. [CrossRef] [PubMed]

30. Deepalakshmi, P.D. Top-down approach of completely sequencing a $4.9 \mathrm{kDa}$ recombinant peptide using quadrupole time-of-flight mass spectrometry. Rapid Commun. Mass Spectrom. 2006, 20, 3747-3754. [CrossRef] [PubMed]

31. Armirotti, A.; Benatti, U.; Damonte, G. Top-down proteomics with a quadrupole time-of-flight mass spectrometer and collision-induced dissociation. Rapid Commun. Mass Spectrom. 2009, 23, 661-666. [CrossRef] [PubMed]

32. Liu, J.; Jiang, J.; Wu, Z.; Xie, F. Antimicrobial peptides from the skin of the Asian frog, Odorrana jingdongensis: De novo sequencing and analysis of tandem mass spectrometry data. J. Proteomics 2012, 75, 5807-5821. [CrossRef] [PubMed]

33. Boeri Erba, E. Investigating macromolecular complexes using top-down mass spectrometry. Proteomics 2014, 14, 1259-1270. [CrossRef] [PubMed]

34. Raynes, J.K.; Vincent, D.; Zawadzki, J.L.; Savin, K.; Logan, A.; Mertens, D.; Williams, R.P.W. Investigation of age gelation UHT Milk. Beverages 2018. submitted for publication.

35. Vincent, D.; Ezernieks, V.; Elkins, A.; Nguyen, N.; Moate, P.J.; Cocks, B.G.; Rochfort, S. Milk Bottom-Up Proteomics: Method Optimization. Front. Genet. 2015, 6, 360. [CrossRef] [PubMed]

36. O'Riordan, N.; Kane, M.; Joshi, L.; Hickey, R.M. Structural and functional characteristics of bovine milk protein glycosylation. Glycobiology 2014, 24, 220-236. [CrossRef] [PubMed]

37. Farrell, H.M., Jr.; Jimenez-Flores, R.; Bleck, G.T.; Brown, E.M.; Butler, J.E.; Creamer, L.K.; Hicks, C.L.; Hollar, C.M.; Ng-Kwai-Hang, K.F.; Swaisgood, H.E. Nomenclature of the proteins of cows' milk-Sixth revision. J. Dairy Sci. 2004, 87, 1641-1674. [CrossRef]

Sample Availability: Samples of the compounds are not available from the authors.

(C) 2018 by the authors. Licensee MDPI, Basel, Switzerland. This article is an open access article distributed under the terms and conditions of the Creative Commons Attribution (CC BY) license (http:/ / creativecommons.org/licenses/by/4.0/). 